\title{
THE POTENTIAL EFFECTS OF EXERCISE-INDUCED CORTISOL RELEASE ON EXECUTIVE FUNCTIONING IN PREADOLESCENTS
}

\author{
A Thesis \\ presented to \\ the Faculty of California Polytechnic State University, \\ San Luis Obispo
}

In Partial Fulfillment

of the Requirements for the Degree

Master of Science in Kinesiology

by

Kory Matthew Bettencourt

January 2018 
(C) 2018

Kory Matthew Bettencourt

ALL RIGHTS RESERVED 
COMMITTEE MEMBERSHIP

TITLE: The Potential Effects of Exercise-Induced Cortisol

Release on Executive Functioning in

Preadolescents

AUTHOR: Kory Matthew Bettencourt

DATE SUBMITTED: January 2018

COMMITTEE CHAIR: Cory Jackson Greever, Ph.D.

Assistant Professor of Kinesiology

COMMITTEE MEMBER: Todd Hagobian, Ph.D.

Associate Professor of Kinesiology

COMMITTEE MEMBER: Kelly Bennion, Ph.D.

Assistant Professor of Psychology and Child

Development 


\begin{abstract}
The Potential Effects of Exercise-Induced Cortisol Release on Executive Functioning in

Preadolescents
\end{abstract}

Kory Matthew Bettencourt

Purpose: Moderate-intensity aerobic exercise (MAE) has been shown to elicit improvements in cognition and subsequent academic performance among preadolescents. Aerobic exercise has also shown to increase cortisol release in response to increasing exercise intensity. However, it is unknown if increased cortisol levels following exercise are related to acute improvements in executive function following a bout of MAE in preadolescents. The purpose of this study was to examine the potential effects of increased cortisol release after acute MAE on attention and working memory among preadolescents. Methods: Eleven preadolescents [6 males, 5 females] volunteered to participate in this study (age $=9.45 \pm 1.03$ ). Participants were randomized in a counterbalanced fashion to 30 minutes of rest or 30 minutes of treadmill MAE (60-70\% HR max). Immediately pre-post each condition, participants completed a cognitive battery consisting of tests of attention (Flanker Test) and working memory (List Sorting Working Memory Test), as well as salivary samples for the analysis of cortisol. Linear Regression models were used to assess significance of covariates. Generalized linear models were used to assess significance of changes in each dependent variable against time, condition, time* condition and change in cortisol. Tukey's HSD post-hoc tests for multiple comparisons were used to assess the effect of condition on working memory, attention, and salivary cortisol. Results: There was a significant effect of condition on working memory $(\mathrm{F}=3.16, p=0.04)$, with no change from pre-post rest $(p=0.93)$ and improving from pre-post exercise $(p=0.04)$. There was no effect of condition on attention or salivary cortisol, most likely due to a small sample size. Multiple linear regression models showed a significant effect of age $(p=0.03)$ and change in cortisol $(p=0.007)$ on working memory. Conclusion: Exercise had a positive effect on working memory, however, we were unable to relate this improvement to changes in salivary cortisol due to a lack of statistical power. This study could provide insight into the physiological effects of increased cortisol release on cognition, specifically in regard to working memory. However, more data are needed to achieve sufficient statistical power to detect these relationships.

Key Words: Moderate-Intensity Aerobic Exercise, Cortisol, Preadolescents, ExecutiveFunctioning, Cognition, Hypothalamic-Pituitary Adrenal Axis, Physical Stress 


\section{ACKNOWLEDGMENTS}

First and foremost, I would like to sincerely thank Dr. Cory Greever for all of the support and help he has provided me throughout the time of this project. Without his guidance and expertise in this area, there is no way I could have finished this project in a timely and professional manner. I would also like to thank Dr. Todd Hagobian and Dr. Kelly Bennion for their input and help with the project. Each of them is leader in their respected field and their contributions to this project were invaluable.

I want to also thank the undergraduate and graduate students who put their time and effort into helping with this project. I am truly grateful to have had such hardworking individuals who were excited and willing to donate their time to this project.

To all the faculty and staff in the Kinesiology Department who helped me throughout this time, I am truly grateful to have been part of such a great department. Your help, support, and funding on this project was truly unbelievable and it helped me out immensely.

The parents and children who volunteered their time to be a part of this research project were truly amazing. Without these individuals and their willingness to be a part of scientific research, this project could not have been possible. For that I thank you.

Finally, I would like to thank my parents for their support during my time in graduate school and for never wavering during the ups and downs of this research project. 


\section{TABLE OF CONTENTS}

Page

LIST OF TABLES $\quad$ ix

LIST OF FIGURES $\quad$ x

CHAPTER

1. INTRODUCTION 1

Statement of Purpose and Research Hypotheses 6

$\begin{array}{ll}\text { Assumptions } & 7\end{array}$

$\begin{array}{ll}\text { Limitations } & 7\end{array}$

$\begin{array}{ll}\text { Definition of Terms } & 7\end{array}$

2. LITERATURE REVIEW 10

$\begin{array}{ll}\text { Objectives } & 10\end{array}$

The Effects of Acute Aerobic Exercise in Relation $\begin{array}{ll}\text { to Executive Functioning } & 10\end{array}$

Neural Mechanisms Effecting Executive Functioning 12

Neuroendocrine Responses and Executive Functioning $\quad 14$

$\begin{array}{lr}\text { Exercise and Cortisol Levels } & 18\end{array}$

The Relationship Between Acute Exercise and 19

Executive Functioning in Relation to Cortisol

$\begin{array}{ll}\text { Summary } & 23\end{array}$

3. METHODS 25

$\begin{array}{ll}\text { Overview and Design } & 25\end{array}$

$\begin{array}{ll}\text { Participants } & 25\end{array}$ 
Screening and Informed Consent/Assent 26

$\begin{array}{lr}\text { Experimental Conditions } & 28\end{array}$

$\begin{array}{ll}\text { Experimental Protocol } & 28\end{array}$

$\begin{array}{ll}\text { Visit } 1 & 29\end{array}$

Visit 2

$\begin{array}{ll}\text { Measures } & 33\end{array}$

Cognitive Tests 33

Inhibition/Attention 33

Working Memory 34

Hormone Analysis $\quad 36$

Salivary Cortisol 36

$\begin{array}{ll}\text { Statistical Methods } & 37\end{array}$

4. RESULTS 39

$\begin{array}{lr}\text { Participants } & 39\end{array}$

$\begin{array}{lr}\text { Generalized Linear Models } & 40\end{array}$

Eriksen Flanker Test of Inhibitory Control
And Attention

List Sorting Working Memory Test 41

$\begin{array}{ll}\text { Salivary Cortisol } & 42\end{array}$

$\begin{array}{ll}\text { Multiple Linear Regression } & 45\end{array}$

Changes in List Sorting Working Memory Test Score 45

Changes in Reaction Time and Response Accuracy 45 
5. DISCUSSION AND CONCLUSION

Discussion

Conclusion

REFERENCES

APPENDICES
A. Informed Consent Form
67
B. Informed Assent Form
70
C. Health Screener
72
D. Child Stress Disorder Checklist (CSDC)
75
E. Exercise Protocol Data Sheet
80
F. RPE Scale for Kids 


\section{LIST OF TABLES}

Table

Page

3.1 Schematic Representation of the Study Protocol

32

4.1 Participant Characteristics

39

4.2 Participant Exercise and Sleep Characteristics

40

4.3 Mean Dependent Variable Values by Condition

42 


\section{LIST OF FIGURES}

Figure $\quad$ Page

2.1 Effect of stress on the HPA-axis and subsequent

glucocorticoid release (Constantini \& Hackney, 2013)

2.2 Salivary cortisol levels before, during, and after exercise

among male children (Del Corral et al., 1994)

2.3 Topographic representation of the P3 component as a

function of session and task (Hillman et al., 2009)

3.1 Representation of Eriksen Flanker Test of Attention on iPad (NIH.gov)

3.2 Examples of One-List and Two-List Sorting Task in the

LSWMT (Tulsky et al., 2014)

3.3 SalivaBio Oral Swab (SOS) used to collect salivary cortisol (Salimetrics)

4.1 Mean List Sorting Working Memory Score by Condition

4.2 Mean Salivary Cortisol Levels by Condition

4.3 Relationship Between Change in LSWMT Score and

Change in Salivary Cortisol by Age 


\section{Chapter 1}

\section{INTRODUCTION}

Academic performance among preadolescents continues to be an important issue to parents, teachers, and schools (Wilson, Olds, Lushington, Petkov, \& Dollman, 2016). Physical activity is a growing area of interest in part because of its potential to improve academic performance through enhancing cognition in children (Castelli, Hillman, Buck \& Erwin, 2007; Centers for Disease Control and Prevention, 2010). However, despite a large body of evidence suggesting the potential cognitive benefits of physical activity, physical education classes and other opportunities for physical activity continue to be cut from schools across the United States (Coe et al., 2006).

An important aspect of cognition is executive functioning, defined as higher level cognitive processes composed of inhibition/attention, updating of working memory, and task-switching (Alvarez \& Emory, 2006). In terms of the type of physical activity that may enhance executive functioning, single bouts of aerobic exercise have been shown to produce acute improvements on executive functioning tasks related to memory and attention in preadolescent children (Alves et al., 2012; Budde et al, 2008; Pontifex et al., 2009; Tsukamoto et al., 2016). Yet, the underlying physiological mechanisms by which aerobic exercise improves executive functions are not well understood in this population. One potential mechanism that could influence the relationship between acute aerobic exercise and cognition is the stress response of the hypothalamic-pituitary-adrenal (HPA) axis. Both psychosocial and physical stressors can activate this neuroendocrine pathway to a degree that may either positively or negatively influence cognitive functioning 
(Ulrich-Lai \& Herman, 2009). Stressors serve as stimuli that have a host of physiological effects including up-regulation of activity in the paraventricular nucleus (PVN) of the hypothalamus. This activation then stimulates secretion of corticotropin releasing hormone $(\mathrm{CRH})$, which acts on the pituitary gland by promoting the release of adrenocorticotropic hormone (ACTH). ACTH then signals the adrenal gland to stimulate the synthesis and release of cortisol (McEwen, 2004). A proposed inverted-u relationship exists between cortisol and cognition, with peak benefits seen at moderate concentrations of cortisol. Conversely, higher and lower levels of cortisol release can signify a state of hyper-arousal or hypo-arousal, which can be detrimental to cognition (Lupien et al., 2005). It is possible that modulation of this neuroendocrine pathway following exercise can mediate the relationship between exercise and aspects of cognitive function, however these relationships are understudied in preadolescent populations (Shields, Bonner, \& Moons, 2015).

Working memory and attention/inhibition are two aspects of cognitive functioning, an important measure of cognitive ability among all ages (Shields, Sazma, \& Yonelinas, 2016). Working memory is defined by the ability to temporarily store information in mind and update or integrate current contents with new information (Shields, Sazma, \& Yonelinas, 2016). Inhibition refers to the ability to inhibit thoughts or responses allowing one to selectively attend to task-relevant information and engage in goal-directed rather than recurrent actions (Shields, Sazma, \& Yonelinas, 2016). Attention, an important aspect of inhibition, is the ability to selectively focus on relevant tasks (Shields, Sazma, \& Yonelinas, 2016). Both working memory and attention tests can serve as valid measures of an individual's ability to direct and re-direct their processing 
resources appropriately (Eysnck \& Keane, 2005). Several studies have investigated the aspects of acute exercise on working memory and attention/inhibition, with many showing positive results among preadolescents (Alves et al., 2012; Budde et al., 2008; Pontifex et al., 2009; Tsukamoto et al., 2016). However, none of these studies have examined the role of HPA-axis modulation on working memory and attention/inhibition in preadolescent children. Therefore, it is important to gain a better understanding of the possible effects of exercise-induced cortisol release on working memory and attention in preadolescents, as it has not been documented among this population.

Exercise intensity may also play a crucial role in the relationship between acute exercise and executive functioning, as different intensities produce different results in terms of cognition (Wang et al., 2013) and cortisol levels (Hill et al., 2008). Previous studies have indicated that as exercise intensity increases, circulating levels of cortisol within the body increase linearly, indicating heightened HPA-axis activity (Hill et al., 2008). As previously mentioned, an inverted-u shape relationship exists among cortisol and working memory in preadolescent children, with higher and lower levels of cortisol being linked to smaller improvements in working memory compared to moderate levels of cortisol release (Lupien et al., 2005). However, the potential mediating role of changes in HPA-axis activity following exercise in improving cognition in preadolescent children remains unknown.

The underlying physiological mechanisms driving the acute benefits of moderate intensity aerobic exercise on executive functioning may be related to the rise in circulating cortisol that occurs after the onset of physical stress. Physical exercise is a known stressor that drastically modifies circulating cortisol levels in humans, increasing 
incrementally as intensity increases (Hill et al., 2008). As previously mentioned, stress induces a state of allostasis which causes a cascade of events, eventually leading to the release of cortisol from the ACTH. It has been proposed that an inverted-u shape relationship exists among cortisol and cognition, specifically pertaining to working memory in preadolescent children (Tsukamoto et al., 2016). This inverted-u may directly correlate with exercise at more vigorous intensities $(80 \%)$, which show very large and immediate increases in cortisol levels, while lighter intensities (40\%) show a very low rise in cortisol levels (Hill et al., 2008). The higher and lower intensities may promote too much or too little a cortisol spike to yield significant increases in cognitive performances following acute exercise. Yet moderate intensities (60-70\%) appear to correlate with the peak of the inverted-u, and may be the ideal intensity for best increasing cognitive performance. Studies have shown that moderate intensity exercise can increase cortisol levels immediately after exercise among preadolescent children (Engel et al., 2014; Del Corral et al., 1994), yet the literature is lacking regarding the mediating role of cortisol on executive functioning after acute bouts of exercise. Only two studies have examined the role of cortisol on working memory after an acute bout of exercise (Budde, PietrassykKendziorra, Bohm, \& Voelcker-Rehage, 2010; Hotting et al., 2016. Yet these studies examined young adults and adolescents respectively, and were unable to find a significant correlation between cortisol, exercise, and working memory. Only one study has examined the effects of cortisol on attention after an acute bout of exercise (Tsai et al., 2014). This study noted increases in reaction time after both high and moderate acute resistance exercise and increases in cortisol levels immediately after exercise among 
subjects between the age of 20 and 29. Still, the effect of cortisol on attention and working memory after acute aerobic exercise is unknown among preadolescents.

One potential theory for improvements in cognition due to exercise-induced cortisol could be due to the increases in cerebral blood flow $(\mathrm{CBF})$ to the brain, creating a cascade of events that may improve cognition. Therefore, it is vital to understand the mediating role of the HPA-axis on working memory and attention after an acute bout of moderate intensity aerobic exercise given that stress-induced neuroendocrine responses cannot be generalized across varying age groups. The developmental stages of the human lifespan alter neuroendocrine activity, therefore, findings from post-pubertal populations cannot be generalized to preadolescents (Constantini \& Hackney, 2013). In fact, prepubertal and post-pubertal populations of the same sex will not typically display the same neuroendocrine responses to exercise (Hackney, McMurray, Judelson, \& Harrell, 2003; Horswill, Zipf, Kien, \& Kahle, 1997). For this reason, it is important to differentiate between hormonal findings among adult populations and preadolescent populations. Cortisol levels typically increase with age (Isurugi et al., 1974; Orentreich, Brind, Rizer, \& Vogelman, 1984; Purifoy, Koopmans, \& Tatum, 1980). These differences between ages can exist at rest, in response to exercise, or after completing an exercise training program (Constantini \& Hackney, 2013). As humans age, they are subjected to progressively higher cortisol levels, which eventually compromises hippocampal integrity and cognitive-dependent hippocampal tasks (Lupien et al., 1998). Preadolescents, less subjected to the long-term effects of glucocorticoids, may not display the same cognitive impairments as older populations. Despite these known age-related differences in neuroendocrine activity, it remains unknown how circulating cortisol levels 
in preadolescents relate to cognitive performance after moderate intensity acute aerobic exercise.

In summary, acute aerobic exercise at moderate intensity has been shown to improve executive functioning in domains such as working memory and attention in preadolescents. It is currently undocumented how changes in cortisol following exercise influence cognition in preadolescents. To our knowledge this is the first study to examine the mediating effects of the HPA-axis on executive functioning after a bout of acute moderate-intensity aerobic exercise among preadolescents.

\section{Statement of Purpose and Research Hypotheses}

The primary purpose of this study is to examine the effects of exercise-induced cortisol release following an acute bout of moderate intensity aerobic exercise on inhibition/attention and working memory in preadolescent children. We hypothesized the following:

When compared to 30 minutes of seated rest, exercising for 30 minutes at $70 \%$ maximum heart rate will exhibit:

1. Increases in response accuracy and reaction time on the Eriksen Flanker Test of attention compared to rest.

2. Increased scores on the List Sorting Working Memory Test.

3. Increased salivary cortisol levels.

4. A positive relationship between increases in salivary cortisol levels and performance on these tests of executive function. 


\section{Assumptions}

This study was based in the following assumptions:

1. It was assumed that the parent(s) of the child participating honestly answered relevant health information pertaining to their child.

2. It was assumed all research assistants followed the protocol outlined in this study.

\section{$\underline{\text { Limitations }}$}

The study was limited by the following factors:

1. Several other physiological mechanisms may play a role in enhanced cognition after exercise. This study only examines one of those potential physiological mechanisms.

2. We did not include a gold-standard method of measuring aerobic fitness or body composition, but we did attempt to address these by measuring moderate-vigorous physical activity and calculating body mass index (BMI).

3. The small sample size is not generalizable to a preadolescent population.

4. Predicted heart rate maximum is not the gold standard method for measuring maximum heart rate.

5. We did not counterbalance the cognitive tests.

\section{$\underline{\text { Definition of Terms }}$}

Acute exercise: Exercise that is brief in duration.

Aerobic: Exercise that is intended to require oxygen.

Attention: Selectively concentrating on a discrete aspect of information. 
Adrenal Cortex: Outer part of the adrenal gland that produces hormones such as cortisol. Adrenal Gland: Endocrine glands that produce a variety of hormones including cortisol. Adrenocorticotrophic hormone (ACTH): A hormone that regulates the levels of cortisol. Cerebral blood flow $(C B F)$ : Blood supply to the brain in a given period of time.

Cognition: The mental process of acquiring knowledge and understanding thought. Congruent: Facing the same direction.

Corticotropin-releasing hormone (CRH): A peptide hormone involved in the stress response

Cortisol: Hormone released by the neuroendocrine system in response to stress. Eriksen Flanker Inhibitory Control and Attention Test: Cognitive test that modulates inhibitory control by having participants attend and respond to a focal stimulus amid an array of other distracting stimuli.

Event Related Potential (ERP): Reflect covert aspects of information processing. Executive Function: General cognitive ability comprised of inhibition, working memory, and shifting.

Hypothalamic Pituitary Adrenal Axis: Major neuroendocrine system component that controls reaction to stress.

Hypothalamus: Region of the forebrain that controls the autonomic nervous system and the pituitary Incongruent: Facing opposite directions.

Inhibition: The ability to attend to task-relevant information while gating out distractors at the same time.

Latency: A time interval between stimulation and response 
List Sorting Working Memory Test: This test assesses working memory by recalling visual stimuli.

Maximum Heart Rate: The upper limit of what your cardiovascular system can handle during physical activity.

Moderate intensity exercise: Physical activity where individual's heart rate is $50-70 \%$ of their maximum

Neuroendocrine system: Maintenance of physiological mechanisms through the hypothalamus P300 Wave: Positive going component observed in the stimulus-locked ERP waveform and is believed to represent the updating of cognitive processes.

Pituitary: Endocrine gland located below the hypothalamus

Physical Stress: Any metabolic, neurological, or endocrinogical changes to the body brought on by physical exertion.

Preadolescents: Stage of human development following early childhood and preceding adolescence Prefrontal Cortex: The cerebral cortex which covers the front part of the frontal lobe. Saliva Oral BioSwab: Salivary cortisol collection method via swab held under the tongue for 1-2 minutes.

Working Memory: Ability to maintain and update information on-line for a short period. 


\section{Chapter 2}

\section{LITERATURE REVIEW}

\section{Objectives}

The objectives of this chapter are to provide an overview of: 1) The effects of acute bouts of aerobic exercise in relation to executive functioning, 2) Neural mechanisms affecting executive functioning 3) neuroendocrine responses in relation to executive functioning in children, specifically in regards to levels of the hormone cortisol, 4) the relationship between exercise and cortisol levels among preadolescent children and 5) the potential mediating effect of increases in cortisol levels following acute exercise improvements in executive functioning.

\section{The Effects of Acute Aerobic Exercise in Relation to Executive Functioning}

It has been well documented that physical activity results in improvements in cognitive performance (Sibley \& Etnier, 2003; Tomporowski, 2003b). Sibley \& Etnier (2003), state in their meta-analysis that effect sizes for improvements in executive functioning are larger in children than in adults, suggesting that children may stand to benefit more cognitively from exercise (Sibley \& Etnier, 2003). Moderate intensity aerobic exercise improves executive functioning among all age groups (Audriffen, Tomporowski, \& Zagrodnik, 2008; Lambourne \& Tomporowski, 2010, Pesce \& Audriffen, 2011; Pesce et al, 2011). Exercise intensity is thought to influence executive functioning in an inverted-u effect, with moderate-intensity eliciting greater improvements than light or high intensity exercise (Brisswalter, Collardeau, \& Rene, 
2002; McMorris \& Hale, 2012). Tests of executive functioning including inhibition, working memory, and task shifting have all shown improvements after a bout of acute physical exercise among preadolescents (Chen et al., 2014; Ellemberg \& St-Louis Deschenes, 2010; Hillman et al., 2009; Lambrick et al., 2016). Still, the underlying physiological mechanisms behind these improvements remain unclear.

Inhibition or attention, refers to the ability to inhibit thoughts or proponent responses in order to selectively attend to task-relevant information and engage in goal directed behavior rather than habitual action (Shields, Sazma, \& Yonelinas, 2016). Several studies have examined the effects of an acute bout of physical activity on inhibition, with most the data indicating short bouts of exercise improve this area of cognition. Hillman et al. (2009), reported increases in response accuracy after a 20minute bout of moderate-intensity treadmill exercise among preadolescent children (Hillman et al., 2009). Ellemberg \& St-Louis Deschenes (2010), demonstrated similar results among 36 7-year olds and 36 10-year olds, reporting improvements in tasks of inhibition after 30 -minutes of ergometer cycling at $63 \%$ of maximum heart rate as compared to a resting control group (Ellemberg \& St-Louis Deschenes, 2010). These results are consistent with research findings in older populations. For example, Kamijo et al. (2009), found improvements in inhibition among 12 older (60-74) and 12 younger (19-25) males after 20 minutes of moderate intensity exercise at 50\% $\mathrm{VO}_{2 \max }$ (Kamijo et al., 2009). The existing literature indicates that acute bouts of moderate intensity exercise can increase performance regarding inhibition among preadolescent children.

In addition to improvements in inhibition, exercise may also transiently improve working memory. Working memory is defined as the ability to temporarily store 
information and update or integrate current contents with new information (Shields, Sazma, \& Yonelinas, 2016). However, the effects of exercise on this aspect of cognition in preadolescents has received little attention. Chen et al. (2014), reported that moderate intensity jogging at $60-70 \%$ of max heart rate for 30-minutes improved three primary aspects of cognitive function, including working memory, among $343^{\text {rd }}$ grade and $345^{\text {th }}$ grade students (Chen et al., 2014). A much larger body of research has focused on understanding acute exercise and working memory among adolescents and adults. Pontifex et al. (2009), found that 30 minutes of aerobic exercise on a treadmill at $60-70 \%$ of $\mathrm{VO}_{2 \max }$ resulted in shorter response times on a working memory test compared to preexercise baseline among 21 young adult participants (Pontifex et al., 2009). In a similar fashion, Soga et al. (2015), also reported improvements in working memory among 55 15-16-year-olds after exercising on a treadmill at $60 \%$ and $70 \%$ maximum heart rate compared to a seated control group (Soga et al., 2015). While the existing literature indicates that working memory can potentially be improved following an acute bout of exercise in adults, these results are less established in preadolescent children. The physiological mechanisms underlying improvements in cognition after exercise also remain unclear among the preadolescent population.

\section{Neural Mechanisms Effecting Executive Functioning}

It stands to reason that transient improvements in executive functioning following exercise are related to the activation of specific areas of the brain that become activated with the onset of physical stress due to increased cerebral blood flow (CBF) (Ide \& Secher, 2000; McAuley, Kramer, \& Colcombe, 2004; Querido \& Sheel, 2007). Bode 
(1991) found that arterial pressures of the cerebral artery increased 103\%-108\% during light physical exercise among 29 children aged 6-17 years of age (Bode, 1991). This increase in blood flow may positively affect specific brain regions such as the prefrontal cortex, a neocortical brain region known for its diverse and flexible behavioral repertoire (Miller \& Cohen, 2001). Exercise-induced increases in CBF in the prefrontal cortex is commonly associated with tasks of executive functions (Roberts, Robbins \&Weiskrantz, 1998). It has been suggested that the activation of the prefrontal cortex due to increases in CBF may play an important role in executive functioning among humans. Diamond (2000), concluded that the prefrontal cortex seems to be an important component of complex cognitive functions (Diamond, 2000). Research has shown that children and adults display significant activation of the prefrontal cortex when performing cognitive tasks such as inhibitory control and working memory (Moriguchi \& Hiraki, 2013). It is possible that heightened activation of the prefrontal cortex following exercise corresponds to improvements in cognitive performance (Byun et al., 2014). A potential mechanism underlying heightened activation of the prefrontal cortex and subsequent improvements in cognition may be due to communication between the prefrontal cortex and the neuroendocrine system. The prefrontal cortex is rich in cortisol receptors (Bennion et al., 2013b). The increase in CBF following exercise may cause a neuroendocrine blood-brain response that increases activation of the prefrontal cortex, thereby increasing executive functioning. More specifically, the Hypothalamic-PituitaryAdrenal axis (HPA-axis), may become activated and increase the release of cortisol into the bloodstream. However, the extent to which the HPA-axis mediates the relationship 
between acute exercise and executive functioning due to increased activation of the prefrontal cortex remains relatively unknown among preadolescents.

Neuroendocrine Responses and Executive Functioning

At the onset of stress, the neuroendocrine pathways begin to set forth physiological responses that trigger the release of certain hormones into circulation. One particularly important stress response pathway is the HPA-axis. After the detection of a perceived threat, the brain enters a state of allostasis, which in turn activates the upregulation of activity in the paraventricular nucleus of the hypothalamus (Killian et al., 2016). This leads to the release of corticotropin releasing hormone (CRH) which acts on the pituitary gland to promote the release of adrenal corticotropin releasing hormone (ACTH); ACTH begins to signal the adrenal gland to stimulate the release of cortisol (McEwen, 2004). Many studies have focused on psychosocial stressors, with far less looking at physiological stress such as exercise, on cortisol levels and executive functioning in preadolescent children. 


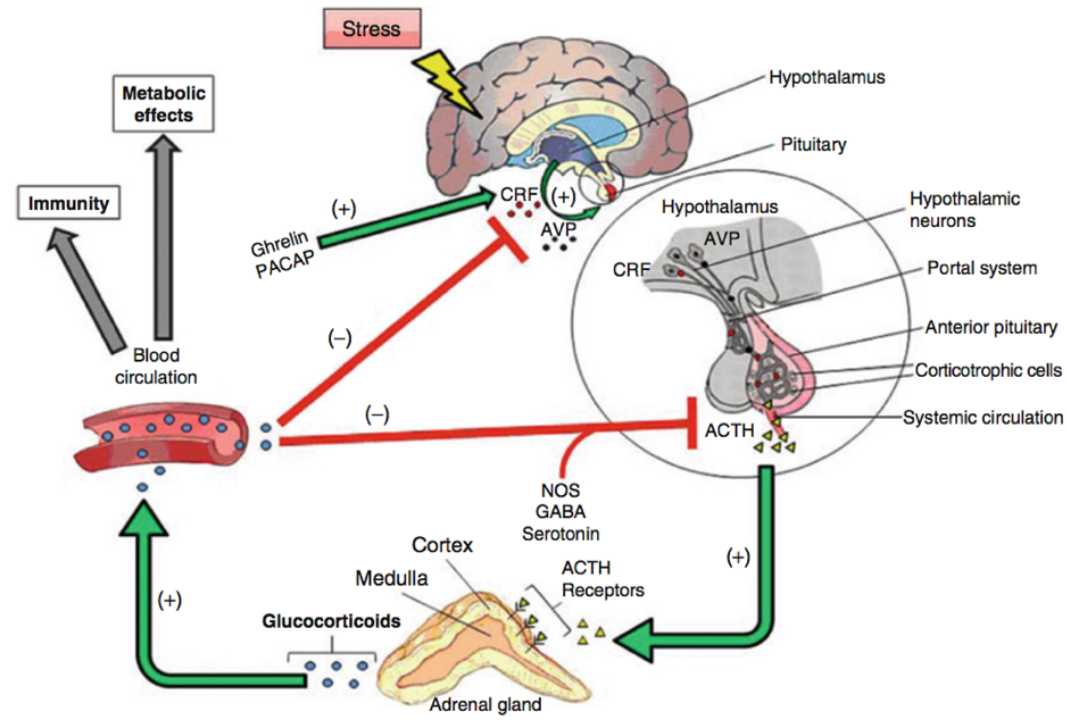

Fig. 2.1. Effect of stress on the HPA-axis and subsequent glucocorticoid release (Constantini \& Hackney, 2013)

Cortisol, released from activation of the HPA-axis, may play an important role in executive functioning. Lupien et al. (2005), demonstrated significant changes in executive functioning in relation to cortisol levels in preadolescent children noting that when cortisol levels were too low or too high, cognition was lower, while moderate amounts of cortisol seemed to elicit the largest improvements in cognition (Lupien et al., 2005). This inverted-u relationship may be essential to understanding how moderate physical stress induces changes in circulating cortisol levels and improves cognition in preadolescent children.

Stress, physiological or psychological, can increase the activity of the HPA-axis, which increases amounts of corticotropin releasing hormone and $\mathrm{ACTH}$, resulting in rises in cortisol amounts (Kirschbaum \& Hellhammer, 1994). A variety of factors influence how much cortisol is released for a given stressor, such as age and maturation. Constanini 
and Hackney (2013), state that different developmental stages in the human life promote different ranges and amounts of hormones, therefore findings cannot be generalized to preadolescents (Constanini \& Hackney, 2013). For instance, pre-pubertal and postpubertal children of the same sex will not display the same hormonal exercise responses or relationships (Hackney, McMurray, Judelson, \& Harrell, 2003; Horswill, Zipf, Kien, \& Kahle, 1997). It is also well known that as humans age, cortisol levels will increase (Isurugi et al., 1974; Orentreich, Brind, Rizer, \& Vogelman,1984; Purifoy, Koopmans, \& Tatum, 1980). As humans age, they are subjected to higher glucocorticoid levels which may slowly compromise hippocampal integrity and cognitive-dependent hippocampal tasks (Lupien et al., 1998). Preadolescents, less subjected to the long-term effect of cortisol, may not display the same impairments when performing cognitive tasks as older populations. Given this information, it is imperative to not generalize how cortisol effects aspects of executive functioning across the lifespan.

Both physical and psychological stress have different effects on various aspects of executive functioning (Shields, Sazma, \& Yonelinas, 2016). High levels of stress are generally thought to impair executive functioning (Arnsten, 2009; Diamond, 2013;

Schoofs et al., 2009; Shansky \& Lipps, 2013), yet it is unlikely stress impairs each aspect of executive functioning in the same fashion (Shields, Sazma, \& Yonelinas, 2016). One potential theory is that stress funnels the most pertinent information relative to the current stressor, causing working memory and cognitive flexibility to be sacrificed in favor of enhanced attention on the task at hand (Shields, Sazma, \& Yonelinas, 2016). Evidence to support this claim come from studies showing that stress impairs working memory (Oei et al., 2006; Schoofs et al. 2008; Shoofs et al., 2009) and cognitive flexibility (Alexander 
et al., 2007; Plessow et al., 2011) but enhances inhibition (Schwabe, 2013). Contrary to this evidence, there are studies that demonstrate stress does not impair or even slightly improves working memory (Duncko et al., 2009; Giles et al., 2014; Luethi et al., 2009; Pesce et al., 2009; Schoofs et al., 2013; Yuen et al., 2009). A potentially important variable that may explain the discrepancy in these studies is the delay between the onset of stress and working memory assessment. Shields et al. (2015), found that exogenous cortisol administration impaired working memory with a short delay between administration and testing, but enhanced working memory with a longer delay (over 1hour post-cortisol administration) among adults (Shields et al., 2015). However, these findings cannot be generalized to preadolescents, and may have yielded different results due to a cortisol being administered exogenously rather than inducing cortisol release endogenously through psychosocial or physical stress stimuli.

The effects of stress on inhibition are less documented. While some studies have shown stress to improve inhibition (Schwabe et al., 2013), other studies have found that stress impairs inhibition (Sanger, et al., 2014). An important mediator between stress and inhibition may be circulating levels of cortisol. Exogenous cortisol administration has been shown to enhance inhibition among adults (Shields et al., 2015), yet due to the effects of maturation on this hormonal axis, the findings cannot be generalized to preadolescents. Several studies examining the effects of physical stress on inhibition among preadolescents have garnered positive results. (Chang et al., 2013; Chen et al., 2014; Ellemberg \& St-Louis-Deschenes, 2010; Hillman et al., 2009). Lambrick et al. (2016), found that 15-minute bouts of either continuous or intermittent treadmill exercise improved reaction time on the Stroop-task among 20 healthy preadolescents (Lambrick et 
al., 2016). Acute physical stress at moderate intensities may be ideal for provoking improvements in inhibitory control. Studies using acute bouts of moderate intensity acute aerobic exercise have shown post-exercise improvements in inhibition (Chang et al., 2013; Chen et al., 2014; Ellemberg \& St-Louis-Deschenes, 2010; Hillman et al., 2009). However, none of these studies examined cortisol release following exercise and its mediating role in improving inhibition. Currently, there is not sufficient evidence to understand if increases in cortisol release enhances aspects of executive functioning after acute bouts of moderate intensity aerobic exercise.

\section{Exercise and Cortisol Levels}

Exercise can be a potent stimulator of circulating cortisol levels among preadolescents (Budde et al., 2010; Del Corral et al., 1994) and adults (Engel et al., 2014; Harris et al., 2016; Hill et al., 2008; Killian et al., 2016). Hill et al. (2008), demonstrated that blood cortisol levels increased among 12 moderately trained men after 30-minutes of aerobic exercise on a cycle ergometer. While circulating cortisol levels increased from rest (-6.6\%) to $40 \%$ of maximal aerobic capacity $(+5.7 \%)$, the most significant increases occurred between $60 \%(+39.9 \%)$ and $80 \%(+83.1 \%)$ of maximal aerobic capacity (Hill et al., 2008). Interpreting these findings in the context of the proposed inverted-u relationship between cortisol and cognition Lupien et al., (2005), the cortisol spike from $60 \%$ to $80 \%$ of maximal aerobic capacity may be inadequate in improving executive functioning. Conversely, the increase in cortisol from rest to $40 \%$ of maximal capacity may not elicit enough of a cortisol response to improve executive functioning. McMorris (2009), reasons that cortisol release modulates arousal by limiting CRH and ACTH, but 
as intensity increases, cortisol is unable to inhibit these hormones, thereby compromising cognitive performance (McMorris, 2009). Del Corral et al., (1994), found that serum cortisol concentrations increased $43 \%$ and $57 \%$ above resting levels at 15 and 30 minutes of exercise at $70 \%$ of maximal capacity in ten healthy preadolescents, which is similar to the $39.9 \%$ increase in cortisol observed at $60 \%$ of maximal capacity in the Hill et al., (2008) study (Del Corral et al., 1994). Therefore, it is possible that moderate intensity exercise triggers an optimal release of cortisol that partially mediates acute improvements in cognition among preadolescents.

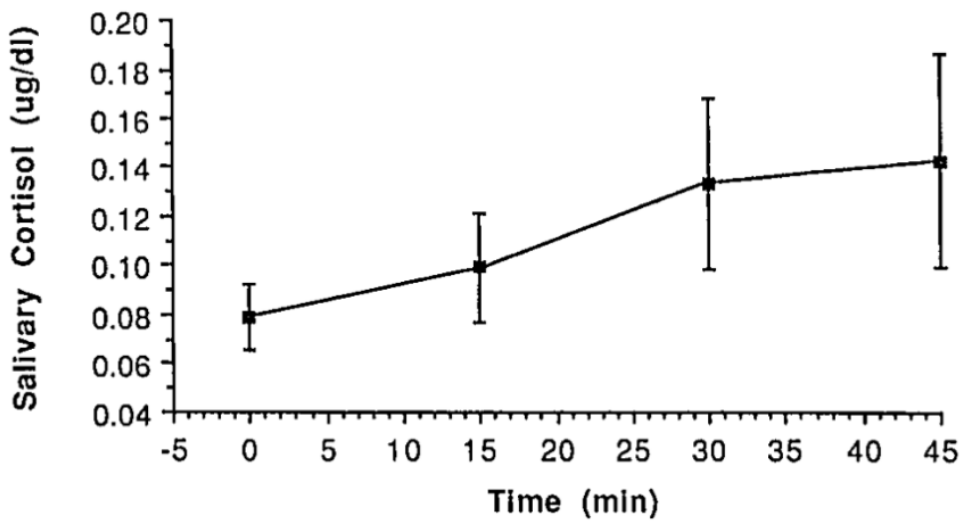

Fig 2.2. Salivary cortisol levels before, during, and after exercise among male children (Del Corral et al., 1994)

The Relationship Between Acute Exercise and Executive Functioning in Relation to

\section{$\underline{\text { Cortisol }}$}

Acute exercise may augment certain brain wavelengths which are indicative of the neuroelectric activity in different regions of the brain. One such is the P300 wave, an 
endogenous component of the event-related potentials (ERP) that occurs approximately 300-800 milliseconds after the onset of a stimulus (Polich et al., 1990). ERP's reflect covert aspects of information processing that provide insight into mechanisms underlying executive functioning (Hillman, Kamijo, \& Scudder, 2011). It is theorized that the P3 wave can index processes involved in the allocation of attention and working memory (Donchin, 1981). The P3 wavelength originates in the central, parietal, and fronto-central regions of the brain, the latter of which includes the prefrontal cortex (Best, 2010). As previously mentioned, activation of the prefrontal cortex plays an important role in executive functioning among children and adults potentially due to an increase in uptake of oxygen supply from a rise in cerebral blood flow after exercise (Budde et al., 2008; Diedrichsen et al., 2007; Ellemberg \& St. Louis-Deschenes, 2010; Serrien et al., 2006, 2007; Tomporowski, 2003a). It is possible that activation of the prefrontal cortex after an acute bout of exercise can trigger increase in P3 wave amplitude, increasing attentional focus and memory. Furthermore, it is possible that increased cortisol release after exercise can activate the cortisol receptor-rich prefrontal cortex, stimulating activation of the P3 wave amplitude, thereby improving cognition. Research has shown that adults who engage in regular aerobic exercise exhibit greater P3 amplitude (Polich \& Lardon, 1997) and P3 latency (Dustman et al., 1990; Hillman et al., 2004). Shorter latency indicates faster cognitive processing speed (Hillman, Kamijo, \& Scudder, 2011). Hillman et al. (2009), found that after an acute exercise session at $60 \%$ of estimated maximum heart rate $\left(\mathrm{HR}_{\max }\right)$ for 20 minutes, preadolescents had greater response in $\mathrm{P} 3$ amplitude which were related to improvements on an academic achievement test (Hillman et al., 2009). Yet no changes in $\mathrm{P} 3$ indices were seen after heavier exercise (Kamijo et., 2006) 
and decreases in P3 amplitude were detected flowing cycling until exhaustion (Kamijo et al., 2004) among older populations. However, the underlying neuroendocrine mechanisms that may link acute exercise and neuroelectrical activity remains unknown. One unifying theory suggests that responses in P3 activity following acute aerobic exercise and subsequent improvements in cognition may be dependent on neuroendocrine regulation, however this theory is untested.

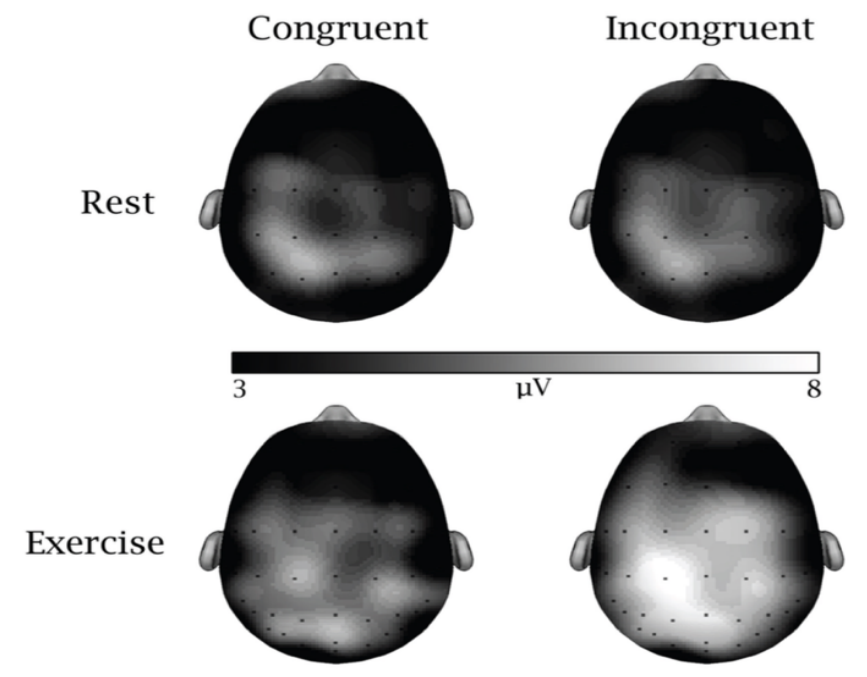

Fig 2.3. Topographic representation of the $\mathrm{P} 3$ component as a function of session and task (Hillman et al., 2009)

While the little research exists regarding the relationship between P3 activity and cortisol, there is some evidence suggesting that cortisol might in fact increase the response of the P3 wavelength. Van Peer, Roelofs, \& Spinhoven (2008), discovered that exogenous cortisol administration resulted in increased response of slow and fast wavelength activity, which included extended analyses of more lateral and posterior electrodes including P3 (Van Peer, Roelofs, \& Spinhoven, 2008). As previously mentioned, a potential reason behind enhanced $\mathrm{P} 3$ wavelength activity could be due to 
increased activation of the prefrontal cortex, a brain region rich in cortisol receptors. Given this information, cortisol may be an important stimulator of $\mathrm{P} 3$ activation which, as Hillman et al. (2009) demonstrated, is activated after acute aerobic exercise among adults and preadolescents, and could be pivotal to the improvements seen in cognition after exercise. However, there are timing differences between these two physiological processes, as increases in cortisol after exercise are seen up to 30 minutes after an acute bout of exercise and P3 responses occur 300 milliseconds after stimulus. These timing differences reflect a general lack of understanding between how these two responses interact after the onset of physical stress. To better understand this connection, studies concurrently examining the effects of acute exercise on cortisol and cognition all together must be conducted.

Very few studies have concurrently examined the impact of acute exercise on cognition and cortisol levels. Tsai et al. (2014), studied the effects of 40 minutes of moderate and high-intensity resistance exercise on inhibitory control and attention and the mediating effects of cortisol (Tsai et al., 2014). The results indicated that inhibitory control and attention improved for both moderate and high intensity resistance exercise conditions, but there were not significant changes in cortisol levels (Tsai, 2014). The two remaining studies examined acute exercise in relation to working memory and the potential mediating of cortisol (Budde et al., 2010; Hotting et al., 2016). Budde et al. (2010), followed 2 experimental groups; one exercising at 50-60\% heart rate max, the other exercising at $70-80 \%$ heart rate max while running around a track for 12 minutes. The results demonstrated cortisol was elevated in the higher intensity group, but not in the lower intensity group, with working memory improving only in the lower intensity 
group (Budde et al., 2010). Finally, Hotting et al. (2016), exposed 81 healthy collegeaged university students to either 30 -minutes of high intensity exercise $(80 \%$ heart rate max), low intensity exercise ( $<57 \%$ heart rate max), followed by a task involving encoding a list of words. The high- intensity exercise group displayed increased cortisol levels after exercise compared to baseline, and recalled a greater number of words 24 hours post-exercise compared to the resting group. However, there was not a significant relationship between cortisol and memory scores (Hotting et al., 2016). There were limitations to these studies that may impact interpretation of the results. First, Tsai et al., (2014) used resistance exercise instead of aerobic, which induces a completely different set physiological responses. Additionally, none of the studies investigated a preadolescent population, meaning it is still unclear as to whether activation of the HPA-axis and the release of cortisol through physical stress can improve cognition among children.

\section{$\underline{\text { Summary }}$}

Improvements in executive functioning following bouts of acute aerobic exercise have been demonstrated in children (Chen et al., 2014; Hillman et al., 2009; Sibley \& Etnier, 2003; Verburgh et al., 2013). Moderate intensity exercise seems to elicit the greatest improvements in cognition, with lower and high intensities being seeming less effective (Chang, Chen, \& Wang, 2011; Chang \& Etnier, 2009). One potential mechanism that may modulate the connection between acute moderate intensity exercise and cognition is the activation of the HPA-axis and the subsequent release of cortisol. It has been proposed that moderate amounts of cortisol may elicit a better cognitive response compared to higher and lower levels, suggesting an inverted-u relationship 
between cognition and cortisol release (Lupien et al., 2005). Therefore, exercise at 60$70 \%$ heart rate max (moderate intensity) may be of greater cognitive benefit to adults and preadolescents. It has been suggested that the transient cognitive benefits observed following moderate intensity aerobic exercise may be due in part to the activation of the P3 wavelength. Cortisol may play an important role in the stimulation of $\mathrm{P} 3$ activation through increased cerebral blood flow to the brain. Yet, to better understand this connection, it is important to concurrently examine the effects of acute exercise on cognition and cortisol in the context of the same study. The current project is the first to examine how activation of the HPA-axis and the subsequent release of cortisol through acute, moderate-intensity aerobic exercise influences working memory and inhibition among preadolescents. 


\section{Chapter 3}

\section{METHODS}

\section{Overview and Design}

The purpose of this study was to examine the effects of 30 -minutes of moderateintensity aerobic exercise on aspects of executive functioning, specifically attention/inhibition and working memory, and the potential mediating role of cortisol release among preadolescent children compared to a resting condition. An experimental, randomized and counterbalanced, within-subject design was used, where participants served as their own control. The conditions were divided into two visits to the Cal Poly Human Performance Laboratory on two separate days, with a one-week washout period separating the two visits. The visits were conducted as follows: Visit 1) Participants were randomized to either an exercise condition or a control condition. Each participant completed the assigned condition (rest or exercise). Visit 2) Participants then switched conditions (rest to exercise, exercise to rest). Hormonal measurements were taken immediately before and immediately after both the resting or exercise condition. Immediately before and after the resting and exercise condition the Eriksen Flanker Test of Attention and the List Sorting Working Memory Test (LSWMT) were administered.

\section{$\underline{\text { Participants }}$}

Eleven preadolescents [6 males, 5 females] enrolled in $3^{\text {rd }}$ to $5^{\text {th }}$ grade were recruited to participate in this study. Participants were recruited from elementary schools of surrounding communities around San Luis Obispo County through email, phone call, flyers, and face-to-face visits with parents and/or school administrators. 
Inclusion criteria included: 1) Children currently enrolled within grades 3rd-5th, 2) Participants attend school within San Luis Obispo County, 3) Children can participate in 30 minutes of moderate aerobic exercise. Exclusion criteria included: 1) Any preexisting heart conditions, 2) any pre-existing lung conditions, 3) any metabolic conditions, 4) emotional or mental disorders, 5) major medical condition that prohibits physical activity, 6) Being left-handed, 7) Spanish speakers, 8) participating in other studies utilizing the same stimuli.

All inclusion and exclusion criteria were reported by a parent of the subject being tested. Informed Consent was obtained from a parent during the 1st visit to the laboratory. The parent(s) were also asked to fill out additional screening forms including a questionnaire regarding socioeconomic status, and a life stress questionnaire. Participants being tested were informed of the risks, benefits, and requirements of the study through an informed assent form that was signed by every child. IRB approval for all protocols and documents was granted by the Human Subjects Committee at Cal Poly.

\section{$\underline{\text { Screening and Informed Consent/Assent }}$}

Two separate documents were handed out to both the parent(s) and the participants of the study during the initial visit to the Human Performance Laboratory. First, an informed consent form was given to the parent(s) who attended the first session with their child. The informed consent provides detailed information to the parent(s) about the research protocols including the potential risks, the purpose of the study, the benefits of the study, confidentiality, and detailed information regarding the exercise protocol and salivary hormonal samples. If the parents felt uncomfortable about any of 
the procedures regarding the research protocol, they were given the right to not sign the consent form without loss of benefits.

An informed assent form was given and read to each child participating in the study. The assent form is written in a language that was understandable to elementary school-aged children. The form provided information to the child about the testing procedures, the risks associated with the test, discomforts they may feel, and individuals they can talk to if they have questions about the research process. If the child felt uncomfortable about any of these procedures, they had the right to forego signing the form and allowed to discontinue their participation in the study without loss of benefits. If the child felt comfortable about all the procedures and signed the form, they became an eligible participant in the study.

Legal guardians were also asked to complete the Child's Stress Disorder Checklist (CSDC). The CSDC questionnaire determines stress over a 2-year period using a points system, with higher scores indicating a higher level of stress. Finally, socioeconomic status (SES) was determined using a trichotomous index based on (a) participation in free and reduced lunch, (b) highest level of education obtained by the mother and father, and (c) number of parents who work full time (Birnbaum et al., 2002). These questions were included in the screening process.

After all forms were signed and participants deemed eligible, the study was explained in more detail to both the parent(s) and the participants. After the protocol was thoroughly explained in detail, the participant began the protocol for the initial visit. 


\section{$\underline{\text { Experimental Conditions }}$}

The experimental conditions consist of: 1) A resting condition, where subjects sit quietly for 30 minutes while watching an age appropriate television show, and 2) An exercise condition where subjects exercised at a corresponding moderate intensity $(70 \%$ HR max) for 30 minutes on a treadmill. During both conditions, salivary cortisol samples were collected immediately before and immediately after the 30 minutes of rest/exercise. Also, immediately after both conditions, subjects completed two separate cognitive tests. Subjects were told to wear comfortable clothes and were constantly monitored, especially during the exercise session. Each participant was told they could stop at any point during any of the procedures if they did not feel comfortable.

\section{$\underline{\text { Experimental Protocol }}$}

Participants completed two different protocols on two separate days with a washout period of 7 days in between the two visits to the Human Performance Laboratory. During Visit 1, after the appropriate forms were filled out and returned, subjects were randomized to either a resting condition or exercise condition. On the following visit, subjects were exposed to the opposite condition. The participant's parent(s) were asked to arrive with their child at the Human Performance Laboratory between the hours of 4:00 pm - 6:00 pm. The parent(s) were instructed to have their child refrain from any sort of physical activity or exercise for at least two hours prior to arrival. They were also asked to have their child fasted for at least two hours prior to testing, and also have them refrain from drinking any liquids other than water. 
Upon arrival to the Human Performance Lab, after appropriate forms were obtained, parent(s) and participants were asked if they had engaged in any physical activity prior to arrival. If they indicated they had, participants were instructed to sit quietly for 30 minutes to allow circulating cortisol levels to return to resting levels. This protocol was also followed upon arrival to the laboratory for the second visit.

Visit 1

During the initial visit, upon arrival to the testing facility, height and weight were measured by a trained research assistant after all forms were filled out and collected. Height was measured by a stadiometer to the nearest $0.1 \mathrm{~cm}$. Weight was determined by digital scale to the nearest $0.1 \mathrm{~kg}$. Height and weight were subsequently used to calculate Body Mass Index (BMI). Following this, the first salivary cortisol sample was collected and immediately labeled and immediately frozen. Following collection of the first saliva sample, participants completed two cognitive tests on an iPad. The first was the Eriksen Flanker Test of Attention and the second was the List Sorting Working Memory Test in that order. After the tests concluded, participants began 30-minute of moderate-intensity exercise or 30-minutes of sitting and watching a television show titled Raising the Giant Dinosaur depending on what condition they were randomized to first. Immediately following completion of their first condition, another salivary cortisol sample was collected. Immediately following this cortisol sample, each subject took the previously mentioned cognitive tests for a second time. Finally, before the participant left, they were fitted with an Actigraph accelerometer on their left wrist to measure physical activity and sleep activity during the one-week washout period. 
The participants assigned to the exercise condition were informed of the protocol and provided detailed instructions of how the exercise condition would be conducted. A heart rate max equation was used to estimate each participant's maximum heart rate. We used this estimation to calculate $70 \%$ maximum heart rate corresponding to moderate intensity. A Polar heart rate monitor was attached to each participant to ensure heart rate stayed within $+/-5 \%$ of $70 \%$ of their max heart rate during the exercise. Participants completed a 3-minute warmup consisting of walking on the treadmill at 1.5 miles per hour. After the warm-up, the participant began their 30 minutes of exercise. Trained research assistants constantly monitored and collected measurements of heart rate after every minute and ratings of perceived exertion (RPE) at 3, 6, 9, 12, 15, 18, 21, 24, and 27 minutes. The Children's RPE Scale was used as it is specifically designed to assess RPE among children using a 1-10 scale which is easier to understand for children. If at any time the participant felt uncomfortable or could no longer continue the exercise due to any sort of pain they could immediately terminate the exercise protocol without loss of benefits.

Visit 2

The protocol for Visit 2 was exactly the same as Visit 1 with only a few differences. First, no time was dedicated to signing forms or collecting anthropometric data, instead a salivary cortisol sample was immediately collected if the participant was rested. If for any reason the participant may have increased arousal levels from exercise or other forms of stress, they were instructed to sit quietly for 30 minutes to allow for circulating cortisol levels to reach a normal, resting level. Second, the protocol stayed 
exactly the same, with the only difference being the participant changing condition. The participants who completed the resting condition were now be assigned to the exercise condition. The participants who completed the exercise condition completed the resting condition. All protocols mentioned in Visit 1 were the same for Visit 2 to ensure accuracy and validity of results. 
Table 3.1. Schematic Representation of the Study Protocol

\begin{tabular}{|c|}
\hline VISIT 1 \\
\hline Informed Consent/Assent \\
\hline Anthropometric Data Collected \\
\hline $1^{\text {st }}$ Salivary Cortisol Sample \\
\hline Collected \\
\hline Cognitive Tests Administered \\
\hline 1. 30-Minutes Aerobic \\
\hline Exercise at $60-70 \% \mathrm{HR}$ max \\
\hline 2. 30-Minutes of Watching \\
\hline T.V \\
\hline $2^{\text {nd }}$ Salivary Cortisol Sample \\
\hline Collected \\
\hline Cognitive Tests Administered \\
\hline Participant fitted with \\
\hline Accelerometer \\
\hline END OF VISIT 1 \\
\hline
\end{tabular}

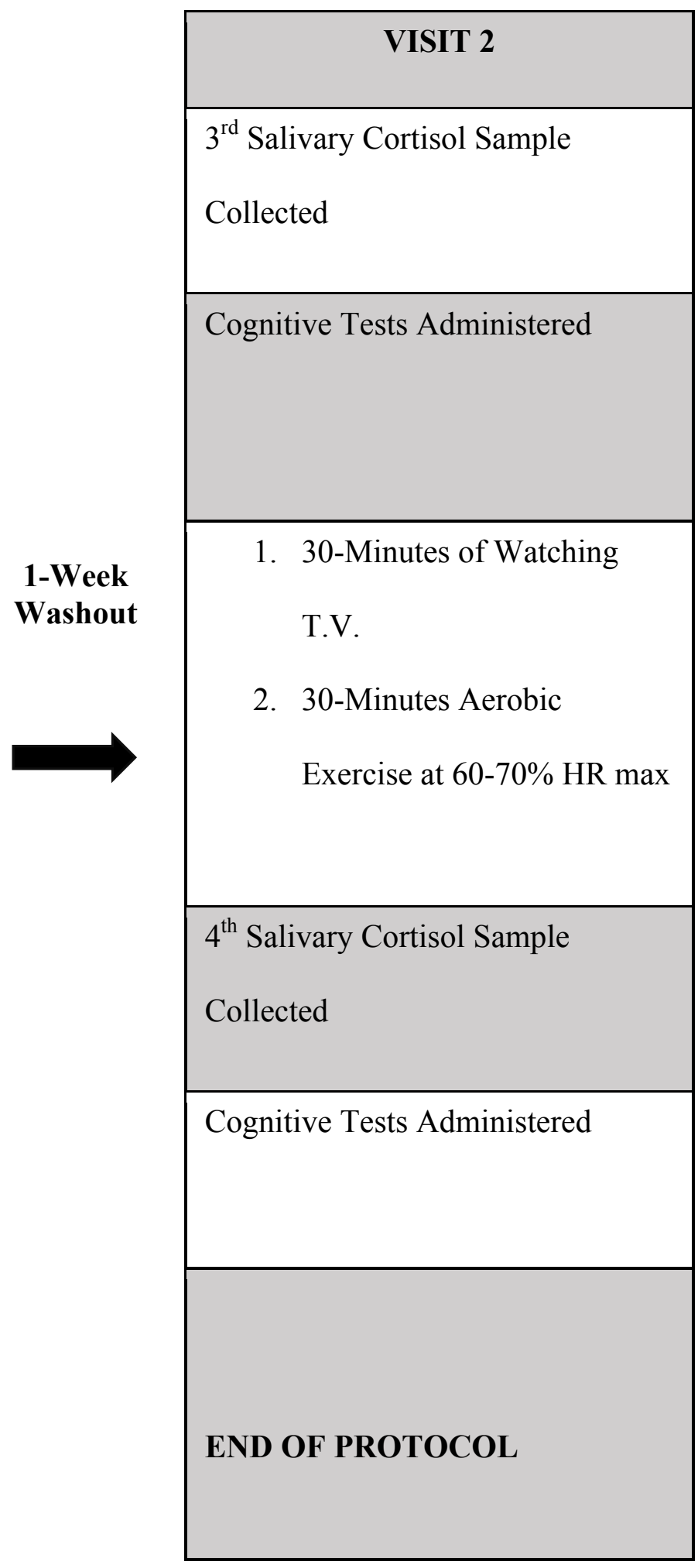




\section{$\underline{\text { Measures }}$}

\section{Cognitive Tests}

Two cognitive tests were administered to every subject immediately before and after each condition. The tests were administered and completed on an iPad in a quiet room while comfortably sitting. The Erikson Flanker Inhibitory Control and Attention Test measured attention and The List Sorting Working Memory Test tested working memory. Each cognitive test was chosen specifically to examine certain aspects of executive functioning that may be affected by exercise induced activation of particular areas of the brain such as the prefrontal cortex. All data were saved to secure file and treated as confidential, with access only available to the primary investigators. If the parents or participants wish to see the results, they were made available only to them.

\section{Inhibition/Attention}

To test inhibition, or attention, The Flanker Inhibitory Control and Attention test was administered during both visits, before and after the 30-minutes of exercise and resting conditions. This test is NIH certified and measures participants' ability to attend to a certain stimulus while inhibiting others. This test consists of a series of arrows presented on the screen, with two arrows on the bottom of the screen pointing left and right. The participant is told to focus only on the middle arrow and press the corresponding arrow for the direction the middle arrow is pointing using the index finger. The test consists of congruent trials, in which arrows all point the same direction $(>>>>>)$ and incongruent trials, where the middle arrow will point in the opposite direction from the other arrows $(>><>>)$. Participants were given four practice trials to 
become familiar with the testing protocol with a research assistant providing directions and answering any questions. After the practice was completed, the participant began the task without help from any research assistants. The participants were told to place their finger at a "home base" button which serves as a constant starting and ending place for each participants index finger. When it was time to respond, they were instructed to answer as quickly and accurately as possible, and then to return their finger back to home base. The accuracy and reaction time of each answer was measured and recorded. Accuracy was measured as the percentage of correct responses out of 20. Reaction time was measured as an average of all 20 times in milliseconds. The test was conducted via iPad and will took between 10-15 minutes to complete.

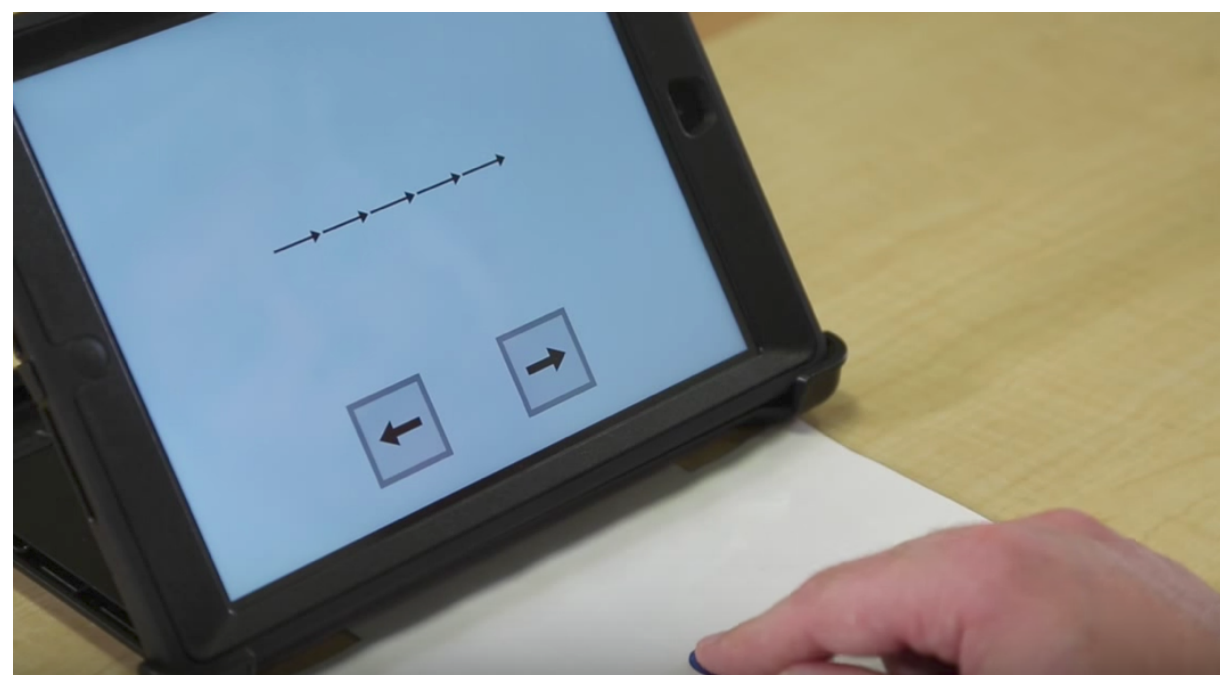

Fig 3.1. Representation of Eriksen Flanker Test of Attention on iPad (NIH.gov)

\section{Working Memory}

The List Sorting Working Memory Test followed the Eriksen Flanker Test of Inhibitory Control and Attention Test and was administered during both visits, 
immediately before and after the 30 minutes of exercise and rest. This test is NIH certified and is designed to test working memory among all age groups. During this test, subjects rely on working memory to remember and re-order a list of pictorial stimuli from smallest to largest in size (e.g., sheep, bunny, elephant). Answers were given orally after a blank screen on the iPad appeared. If an incorrect response was recorded, participants received another chance to re-order the same amount of different pictorial stimuli. If this answer was incorrect, the test proceeded to the two-list items. If two incorrect responses in a row were recorded during the two-list items, the test was over. One-list items have participants re-order one category of lists in size order from smallest to biggest (animals only or food only). Two-list items have participants re-order two different categories in size order from smallest to biggest (food and animals). As the test progressed, one more image would be added to the list, making it more difficult to remember and re-order all of the items. The highest number of images a participant could re-order was seven. Figure 3.2 shows one-list and two-list items with the correct answers. After two initial practice trials with a trained research assistant, participants began the test. This test was monitored by a research assistant, recording correct or incorrect responses while also conducting the examination by following the procedures listed in the NIH Toolbox Manual. This test was also administered on an iPad and uses a keyboard to register answers from the participants. The test took 10-15 minutes to complete. 


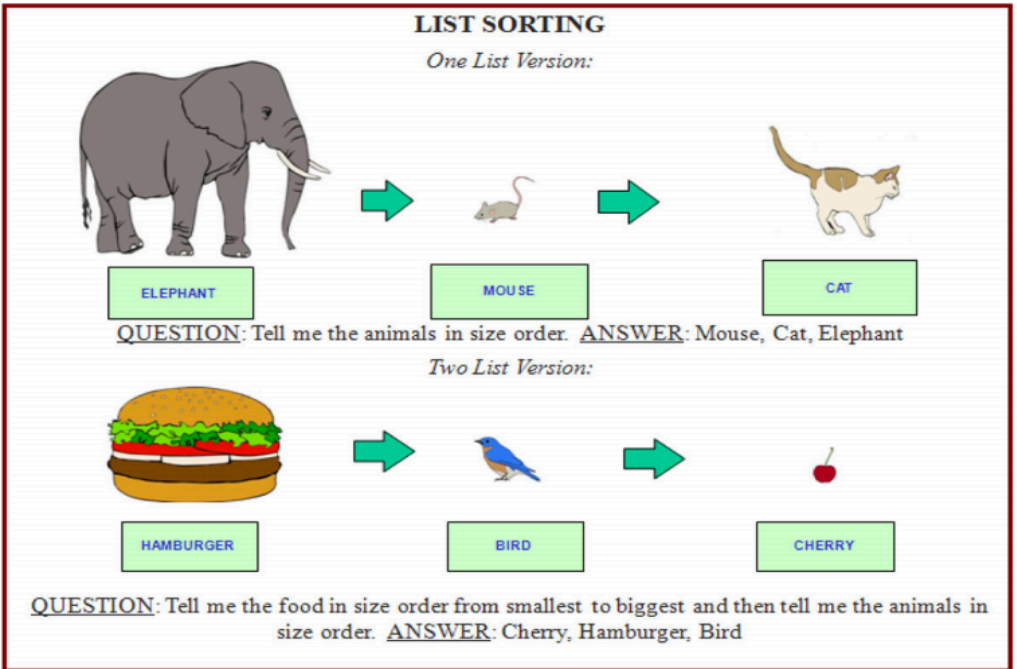

Figure 3.2. Example of One-List and Two-List Sorting Task in the LSWMT (Tulsky et al., 2014)

\section{$\underline{\text { Hormone Analysis }}$}

\section{Salivary Cortisol}

To investigate the impact of acute moderate intensity aerobic exercise on HPAaxis activation, salivary cortisol was measured to see if there were significant differences after exercise as compared to after a resting condition, and to determine if these potential differences mediated changes in executive functioning. Salivary cortisol was collected through SalivaBio Oral Swab (SOS) Saliva Collection Method. A trained research assistant collected the sample through oral swab. The average maximum to be collected is $2 \mathrm{~mL}$. This method of collection is recommended for adults and children ages 6 and up. The swab was placed under the tongue for 1-2 minutes and then removed and placed into the swab tube for later analysis. If the participant felt uncomfortable with a research assistant collecting the sample, they could put the swab under their own tongue under supervision of a research assistant to ensure accurate collection. 
Immediately after the collection, the samples were labeled and then frozen at or below -20 degrees Celsius in a freezer. The samples were shipped to Salimetrics for hormone analysis by trained lab technicians assuring accurate and valid inspection. The samples contained no identifying personal information, and only primary investigators had access to them. Figure 3.3 shows the tube and swab used in the collection of salivary cortisol.

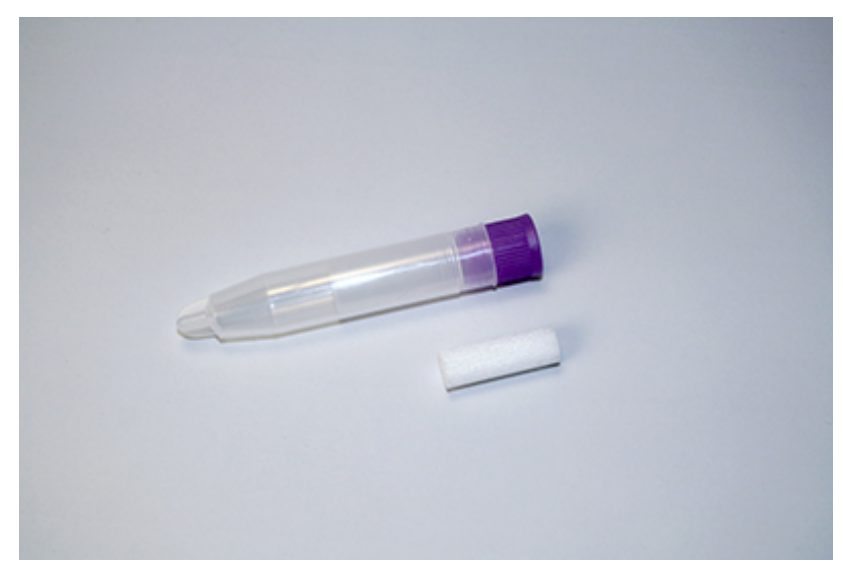

Figure 3.3. SalivaBio Oral Swab (SOS) used to collect salivary cortisol (Salimetrics).

\section{$\underline{\text { Statistical Methods }}$}

All statistical analyses were performed using JMP Stats version 13 (SAS Institute, Cary, NC) statistical software. The effects of time, condition, and time*condition on our executive function variables and cortisol levels were determined using a Generalized Linear Model. Tukey's post-hoc analysis was used to determine differences between conditions and time points. The potential mediating effects of changes in cortisol on changes in dependent variables were tested using a Multiple Linear Regression Analysis with a backward selection technique. Backward selection technique was performed by deleting the least significant variable until only significant covariates are left. This regression technique was used to test the relationship between change scores (pre-post) in 
our executive function variables with relevant covariates and changes in cortisol. Final models revealed significant covariates for each dependent variable as follows: Working Memory [Cortisol change, age]; Reaction Time [Average Sleep Time, ModerateVigorous Physical Activity].

A minimum sample size of $n=39$ was calculated to achieve a statistical power $80 \%$. This value was calculated by the Cal Poly Statistics Department utilizing the Hillman et al., (2009), and Del Corral et al., (1994) studies as references. A significance level of $\mathrm{p}<0.05$ was used for all comparisons. 


\section{Chapter 4}

\section{RESULTS}

\section{$\underline{\text { Participants }}$}

Participant characteristics for age (years), height $(\mathrm{cm})$, weight $(\mathrm{kg})$, body mass index (BMI), child stress disorders checklist score (CSDC), and socioeconomic status (SES) were reported in means and standard error in table 4.1. Participant exercise and sleep characteristics were reported in means and standard error in table 4.2.

Table 4.1. Participant Characteristics

\begin{tabular}{lccc} 
Variable & All Participants & Females (n=5) & Males (n=6) \\
\hline Age & $9.45(1.03)$ & $9.40(0.89)$ & $9.50(1.22)$ \\
Height $(\mathrm{cm})$ & $138.72(6.76)$ & $142.10(7.30)$ & $135.91(5.29)$ \\
Weight $(\mathrm{kg})$ & $31.69(5.87)$ & $34.45(6.53)$ & $29.39(4.57)$ \\
BMI (PCTL)* & $52.40(1.58)$ & $64.10(1.57)$ & $39.40(1.57)$ \\
CSDC * & $2.18(2.77)$ & $2.20(3.90)$ & $2.03(1.79)$ \\
SES & $3.00(0.00)$ & $3.00(0.00)$ & $3.00(0.00)$ \\
\hline * Percentile & & \\
$* *$ Child Stress Disorder Checklist (CSDC) & & \\
\hline
\end{tabular}

BMI percentile was calculated using the Center for Disease Control calculator (CDC.gov). SES was measured using a trichotomous index based on (a) participation in free and reduced lunch, (b) highest level of education obtained by the mother and father, and (c) number of parents that work fulltime (Birnbaum et al., 2002). Score ranges were from 1 to 3, with higher values representing higher SES. In this study, all participants scored 3, which indicated high SES across the entire sample size. 
Table 4.2. Participant Exercise and Sleep Characteristics

\begin{tabular}{lrrr}
\hline Variable & All Participants & Females & \multicolumn{1}{c}{ Males } \\
\hline Mean Exercise HR & $136.38(7.85)$ & $138.24(6.76)$ & $134.85(8.95)$ \\
RPE* & $5.40(1.51)$ & $5.17(1.36)$ & $5.60(1.74)$ \\
Avg. Sleep Time (min) & $478.23(66.13)$ & $492.47(57.50)$ & $466.45(75.72)$ \\
MVPA (\%)** & $13.98(5.64)$ & $16.33(5.88)$ & $12.02(5.09)$ \\
& & \\
$\begin{array}{l}* \text { Rating of Perceived Exertion } \\
* * \text { Moderate to Vigorous Physical Activity Percentage [time spent in percentage per day } \\
\text { during one-week washout period] }\end{array}$ & \\
\hline
\end{tabular}

Covariates (age, BMI, SES, CSDC score, MVPA\%, and average sleep time) were tested for significance by using a backward selection regression analysis. A complete overview of the mean values for each dependent variable by condition from this study can be seen in Table 4.3.

\section{$\underline{\text { Generalized Linear Models }}$}

Generalized Linear Models were used to determine the effect of time, condition, and time*condition on our executive function variables. The Generalized Linear Model was utilized to determine potential differences before and after exercise among our dependent variables.

\section{Eriksen Flanker Test of Inhibitory Control and Attention}

It was hypothesized that, when compared to the resting condition, the exercise condition would decrease participants' average reaction time and increase the number of correct responses on the task. It was also hypothesized that an increase in salivary cortisol 
would correspond to these potential decreases in reaction time and increases in correct responses.

There was no effect of time [pre, post] $(p=0.8)$, condition [rest, exercise] $(p=0.3)$, or cortisol $(p=0.9)$ on reaction time. There was also no effect of an interaction between time and condition $(p=0.6)$. Similarly, there was no effect of time $(p=0.4)$, condition $(p=0.9)$, cortisol $(p=0.8)$, or the interaction of time and condition $(p=0.2)$ on reaction accuracy. These findings suggest that neither exercise or cortisol significantly affected these two aspects of the Eriksen Flanker Task of attention.

\section{List Sorting Working Memory Test}

It was hypothesized that, when compared to the resting condition, the exercise condition would increase participants' scores on the LSWMT. It was also hypothesized that increases in salivary cortisol would correspond to these potential increases in test scores.

A significant effect of time [pre, post] $(p=0.04)$ on change in LSWMT scores was observed after 30 minutes of moderate intensity aerobic exercise. However, similar to the Eriksen Flanker Task, there was no significant effect of cortisol change on LSWMT score $(p=0.9)$; and no significant effect of condition [rest, exercise] $(p=0.09)$, or the interaction of time*condition $(p=0.1)$. This finding indicates that moderate intensity aerobic exercise for 30 minutes improved working memory and retention among preadolescents, which is congruent with previous findings. Yet these improvements in test scores cannot be correlated to increases in salivary cortisol levels. The results from the LSWMT can be seen in figure 4.1 . 


\section{Salivary Cortisol}

It was hypothesized that, when compared to the resting condition, the exercise condition would increase participants' salivary cortisol levels.

There was no significant effect of time [pre, post] $(p=0.3)$, condition [rest, exercise] $(p=0.4)$, or time* condition interaction $(p=0.6)$ on change in salivary cortisol levels, suggesting exercise did not significantly increase cortisol levels enough to correlate with changes in Eriksen Flanker Task scores or LSWMT scores. Although exercise did significantly increase the change in LSWMT scores, we were unable to say that is was due to increases in salivary cortisol, suggesting cortisol had no significant effect as a mediating variable on both the Eriksen Flanker Task and the LSWMT. The relationship between condition and cortisol level can be seen in figure 4.2.

Table 4.3. Mean Dependent Variable Values by Condition

\begin{tabular}{lcccc}
\hline Variable & Pre-Rest & Post-Rest & Pre-Exercise & Post-Exercise \\
\hline $\begin{array}{l}\text { Flanker (RA) } \\
(\text { Out of 20) }\end{array}$ & $20.00(0.00)$ & $19.91(0.30)$ & $19.82(0.40)$ & $19.91(0.30)$ \\
$\begin{array}{l}\text { Flanker (RT) } \\
(\mathrm{ms})\end{array}$ & $0.89(0.25)$ & $0.85(0.16)$ & $0.81(0.15)$ & $0.82(0.14)$ \\
$\begin{array}{l}\text { LSWMT Score } \\
(\text { Out of 26) }\end{array}$ & $17.09(3.30)$ & $17.63(2.62)$ & $16.55(3.98)$ & $19.27(2.94)$ \\
$\begin{array}{l}\text { Cortisol } \\
(\mu \mathrm{g} / \mathrm{dL})\end{array}$ & $0.27(0.15)$ & $0.29(0.19)$ & $0.27(0.16)$ & $0.38(0.39)$ \\
\hline $\begin{array}{l}\text { RT }=\text { Reaction Time } \\
\text { RA }=\text { Response Accuracy }\end{array}$ & & & \\
\hline
\end{tabular}




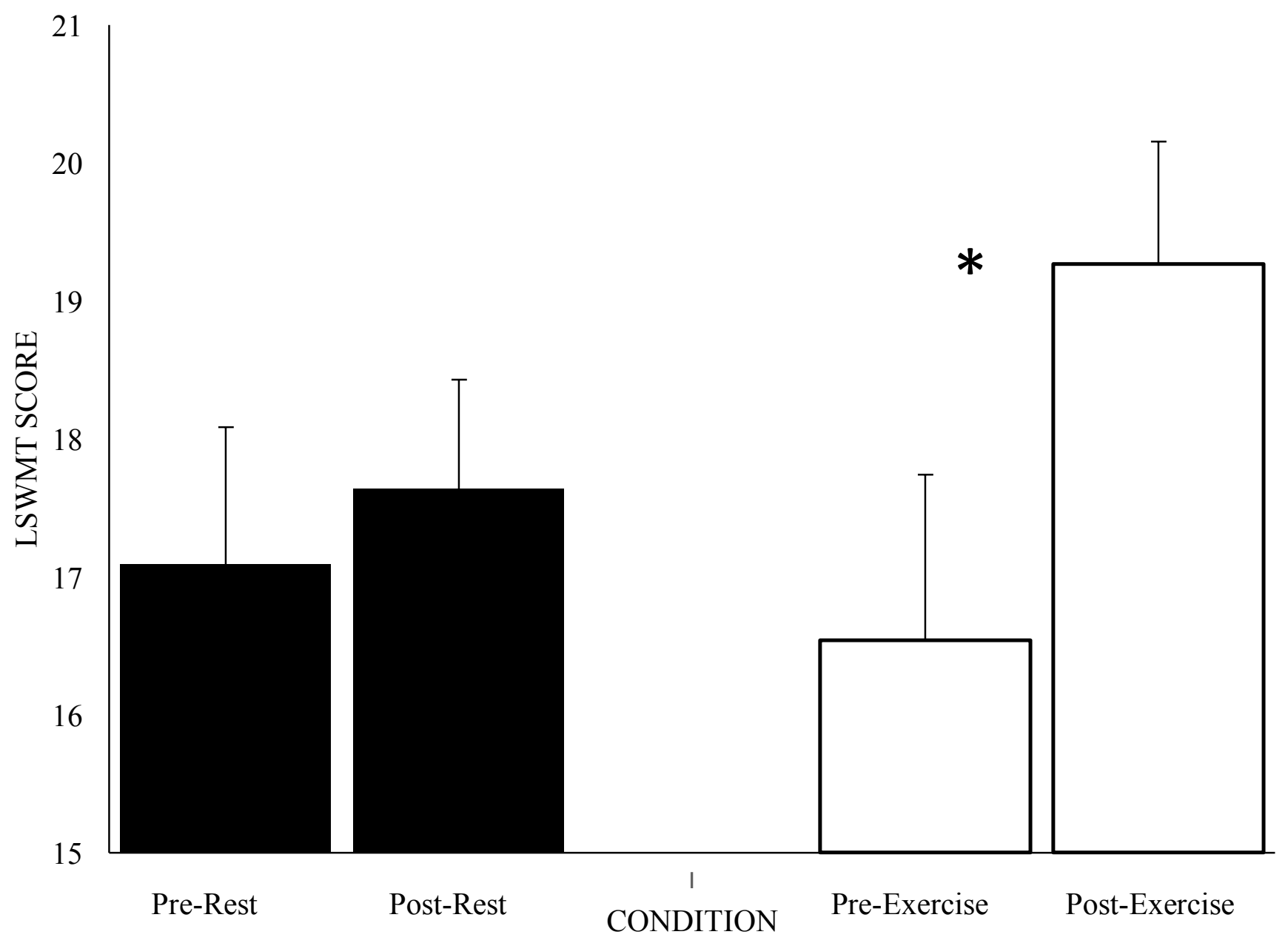

Figure 4.1. Mean List Sorting Working Memory Test Scores by Condition $*_{p}<0.04$ 


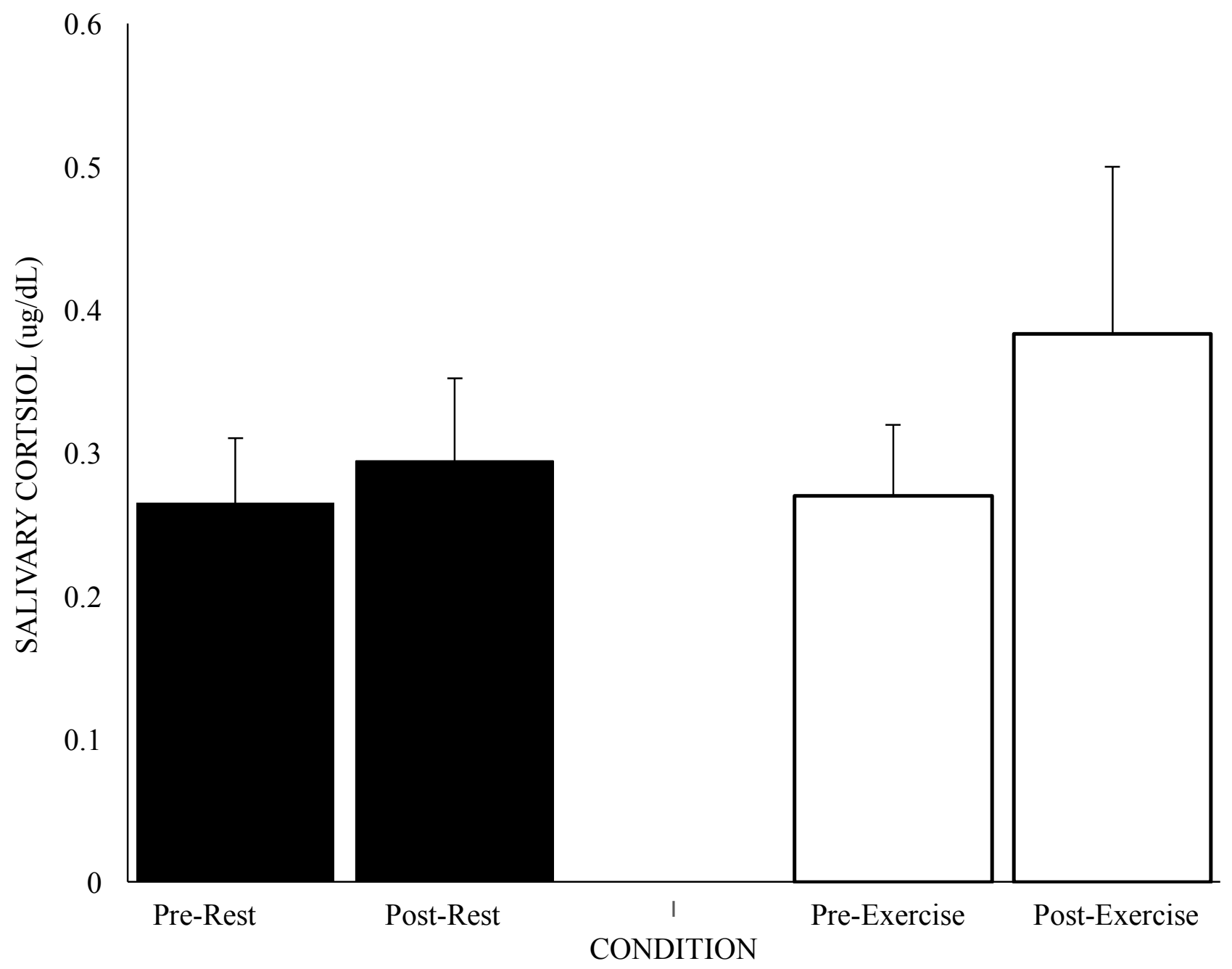

Figure 4.2. Mean Salivary Cortisol Levels by Condition 


\section{Multiple Linear Regression}

To understand which covariates had an effect on the change in our dependent variable scores, Multiple Linear Regression models were run with a backward selection technique to identify significant covariates. Backward selection technique was performed by deleting the least significant variable until only significant covariates are left. It was hypothesized that changes in executive function scores would correlate to changes in salivary cortisol, lending to the idea that cortisol mediates potential improvements in executive function after an acute bout of exercise.

\section{Changes in List Sorting Working Memory Test Score}

After backward selection technique was applied to the change in LSWMT, there was a significant correlation between two covariates; change in salivary cortisol $(p=0.007)$ and age $(p=0.03)$. Figure 4.3 shows the relationship between change scores on the LSWMT and change in salivary cortisol levels separated by age.

\section{Changes in Reaction Time and Response Accuracy}

There were no significant correlations between changes in reaction time and changes in cortisol. However, there was a significant correlation between changes in reaction time and two covariates; time spent in moderate to vigorous physical activity $(p=0.02)$ and average sleep time $(p=0.0001)$. No covariates showed significant correlations to change in response accuracy. 


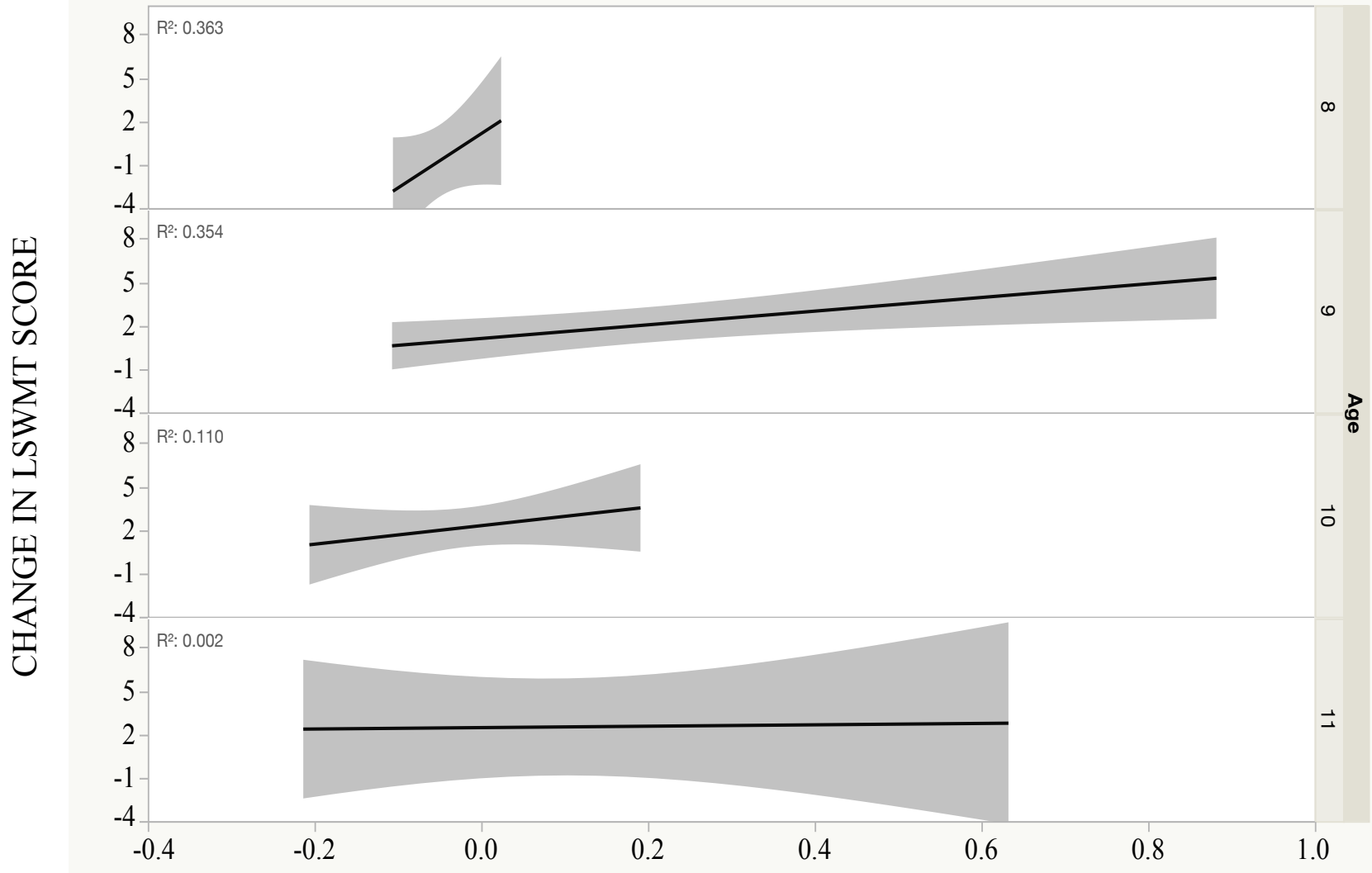

CHANGE IN SALIVARY CORTISOL $(\mu \mathrm{g} / \mathrm{dL})$

Figure 4.3. Relationship Between Change in LSWMT Score and Change in Salivary Cortisol by Age 


\section{Chapter 5}

\section{DISCUSSION AND CONCLUSION}

\section{$\underline{\text { Discussion }}$}

The purpose of the present study was to examine to potential effects of exerciseinduced cortisol release on two aspects of executive functioning - attention and working memory - among preadolescents. It was hypothesized that, when compared to the resting condition, 30 minutes of moderate intensity aerobic exercise would elicit greater: increases in salivary cortisol immediately after the exercise; improvements in reaction time (RT) and reaction accuracy (RA) scores on the Eriksen Flanker Task; and increases in List Sorting Working Memory Test scores. We also hypothesized that there would be a positive relationship between increases in cortisol and increases in executive function scores, indicating a potential mediating relationship of cortisol between acute aerobic exercise and executive functioning. Results suggest that 30 minutes of moderate intensity exercise may increase working memory, as these test scores were significantly increased following the bout of acute exercise compared to a resting condition. Yet we cannot say that these increased test scores corresponded to an increase in salivary cortisol after an acute bout of exercise due to lack of significance. No effect of exercise or cortisol was seen on reaction time or response accuracy.

With the onset of stress, the neuroendocrine system activates a series of hormonal pathways, one such being the activation of the HPA-axis. In response to stress, the HPAaxis sets forth a cascade of physiological events that eventually lead to an increase in cortisol output into the bloodstream (McEwen, 2004). Previous literature suggest that 
exercise increases cortisol release linearly as exercise intensity increases (Hill et al., 2008). Furthermore, exercise has shown exercise increases cortisol levels among preadolescents (Del Corral et al., 1994; Budde et al., 2010). Our findings show that there was no significant increase in salivary cortisol levels after an acute bout of exercise among our preadolescents population. The discrepancy in results could be due to our small population size, or a workload that was not intense enough to elicit such increases, yet further research is warranted to fully understand the relationship between exercise and cortisol levels among preadolescents.

There were no differences observed among reaction time or reaction accuracy, two aspects of attention tested by the Eriksen Flanker Test, after the exercise condition. This is in disagreement with previous research findings indicating moderate intensity aerobic exercise decreases reaction time and increases reaction accuracy in tests of attention and inhibition among preadolescents (Hillman et al., 2009; Ellemberg \& StLouis Deschenes, 2010; Chen et al., 2014). The reasoning behind these differences in results are unknown.

Perhaps the most significant finding from this study was the significant increase in List Sorting Working Memory Test Scores after an acute bout of aerobic exercise (19.28) compared to rest (16.55). Previous research has also demonstrated improvements in working memory after an acute bout of exercise among preadolescents (Chen et al., 2014; Pontifex et al., 2009; Soga et al., 2015). However, none of these studies used the LSWMT as a method for their measurements of memory, meaning this study may highlight the validity of such a measurement for future studies. Our regression analysis showed there was a significant correlation between change in LSWMT score, change in 
salivary cortisol, and age, lending to the idea that increased cortisol after acute bouts of aerobic exercise may enhance executive functions. One potential theory supporting this idea could be the activation of the prefrontal cortex and subsequent activation of the P3 wavelength. With the onset of exercise, there is an increase in cerebral blood flow and subsequent oxygen uptake by the brain (Budde et al., 2008; Diedrichsen et al., 2007; Ellember \& St. Louis-Deschenes, 2010; Serrien et al., 2006, 2007; Tomporowski, 2003a). The prefrontal cortex, an important brain component for memory consolidation, is rich in cortisol receptors (Bennion et al., 2013b), lending to the idea that an increase in cortisol may increase activation of the prefrontal cortex. Hillman et al. (2009), showed that acute exercise enhances P3 wavelength activation among preadolescents, yet it is still unclear as to how the activation of these wavelengths improve aspects of executive functioning such as attention and working memory. Age was also correlated to change in LSWMT scores and change in cortisol, with nine and ten-year old participants seeing the most linear trend among cortisol and working memory changes, likely due to the fact that this age range made up a majority of the sample size of this study.

Although this study was unable to correlate salivary cortisol levels to improvements in test attention, and saw modest correlations between cortisol levels and working memory, it is still unknown as to what extent increases in the HPA-axis have on executive functions. As previously mentioned, one unifying theory that connects exercise to improvements in cognition is through increases in cerebral blood flow and oxygen uptake by areas of the brain such as the prefrontal cortex (Ide \& Secher, 2000; McAuley, Kramer, \& Colcombe, 2004; Querido \& Sheel, 2007). Activation of the prefrontal cortex seems to be an important component of complex cognitive functions (Diamond, 2000). 
Hillman et al. (2009), found that activation of the P3 wavelength, which originates in specific regions of the brain, including the prefrontal cortex, was associated with significant improvements in tests of attention. Because the prefrontal cortex is known to be rich in cortisol receptors (Bennion et al., 2013b), it is still possible that increases in cerebral blood-flow and subsequent increases in cortisol to the prefrontal cortex may activate the P3 wavelength, thereby increasing specific components of executive functions. This study was unable to prove this theory, yet further research with larger samples sizes are needed in order to fully understand the role of the HPA-axis and subsequent cortisol release on aspects of cognition after an acute bout of exercise among preadolescents.

There were several limitations to this study. First, there are several physiological mechanisms that may play a role in enhanced cognition after exercise, this study only focused on cortisol. Second, this study had a small sample size $(n=11)$, which may have been the reason for the discrepancy in these results compared to the existing body of literature. For this reason, this sample size is not generalizable to a preadolescent population as a whole. Third, the parent of the participant was told of restrictions before arriving to the laboratory, yet it is unknown if these recommendations were followed strictly enough. For instance, the parent was told to have the child refrain from physical activity at least two hours prior to the laboratory visit, yet what the child did at school may have differed from our recommendations. Fourth, predicted heart rate maximum is not the gold standard for measuring maximum heart rate. While this equation is convenient, maximal tests of oxygen uptake, $\mathrm{VO}_{2} \max$ would be more precise at measuring specific values for heart rate maximum for individual participants. Fifth, we 
did not include a measure of IQ or other academic competency tests that would have allowed us insight into where these participants were academically compared to national averages. Sixth, we did not counterbalance the cognitive tests, which may have had an effect on test scores due to the timing of when these tests were taken after each condition. Counterbalancing these would have controlled for this factor. Finally, we set our exercise intensity between $60-70 \%$ of $\mathrm{HR}_{\max }$, yet this may have not been a high enough intensity to see significant increases in salivary cortisol.

\section{$\underline{\text { Conclusion }}$}

In conclusion, an acute bout of aerobic exercise at $60-70 \%$ of heart rate max improved scores on a test of working memory, compared to a resting condition, which showed no such improvements. There were no significant increases in salivary cortisol levels after the acute bout of exercise compared to the resting condition, yet this may be due to a lack of statistical power due to a small sample size. Our regression analysis revealed a correlation among changes in LSWMT, changes in salivary cortisol, and age. This finding suggests that there may be a potentially strong correlation between working memory and increases in cortisol levels, yet further research is warranted to fully understand these relationships. Tests of attention yielded no significant results in regard to reaction time and response accuracy. This study provides further evidence to an existing body of literature that suggests preadolescents may be able to improve memory and retention after an acute bout of moderate intensity aerobic exercise, which may subsequently improve academic performance. 


\section{REFERENCES}

Alexander, J. K., Hillier, A., Smith, R. M., Tivarus, M. E., \& Beversdorf, D. Q. (2007). Betaadrenergic modulation of cognitive flexibility during stress. Journal of cognitive neuroscience, 19(3), 468-478.

Alvarez, J. A., \& Emory, E. (2006). Executive function and the frontal lobes: a meta-analytic review. Neuropsychology review, 16(1), 17-42.

Alves, C. R., Gualano, B., Takao, P. P., Avakian, P., Fernandes, R. M., Morine, D., \& Takito, M. Y. (2012). Effects of acute physical exercise on executive functions: a comparison between aerobic and strength exercise. Journal of sport \& exercise psychology, 34(4), 539-549.

Ando, S., Yamada, Y., \& Kokubu, M. (2010). Reaction time to peripheral visual stimuli during exercise under hypoxia. Journal of Applied Physiology, 108(5), 1210-1216.

Anish, E. J. (2005). Exercise and its effects on the central nervous system. Current sports medicine reports, 4(1), 18-23.

Arnsten, A. F. (2009). Stress signalling pathways that impair prefrontal cortex structure and function. Nature Reviews Neuroscience, 10(6), 410-422.

Audiffren, M., Tomporowski, P. D., \& Zagrodnik, J. (2008). Acute aerobic exercise and information processing: energizing motor processes during a choice reaction time task. Acta Psychologica, 129(3), 410-419.

Bennion, K. A., Ford, J. H., Murray, B. D., \& Kensinger, E. A. (2013). Oversimplification in the study of emotional memory. Journal of the International Neuropsychological Society, 19(09), 953-961. 
Bennion, K. A., Steinmetz, K. R. M., Kensinger, E. A., \& Payne, J. D. (2013). Sleep and cortisol interact to support memory consolidation. Cerebral Cortex, bht255.

Best, J. R. (2010). Effects of physical activity on children's executive function: Contributions of experimental research on aerobic exercise. Developmental Review, 30(4), 331351.

Bode, H. (1991). Cerebral blood flow velocities during orthostasis and physical exercise. European journal of pediatrics, 150(10), 738-743.

Brisswalter, J., Collardeau, M., \& René, A. (2002). Effects of acute physical exercise characteristics on cognitive performance. Sports medicine, 32(9), 555-566.

Buchanan, T. W., \& Lovallo, W. R. (2001). Enhanced memory for emotional material following stress-level cortisol treatment in humans. Psychoneuroednocrinology, 26(3), 307-317

Budde, H., Pietrassyk-Kendziorra, S., Bohm, S., \& Voelcker-Rehage, C. (2010). Hormonal responses to physical and cognitive stress in a school setting. Neuroscience letters, 474(3), 131-134.

Budde, H., Voelcker-Rehage, C., Pietraßyk-Kendziorra, S., Ribeiro, P., \& Tidow, G. (2008). Acute coordinative exercise improves attentional performance in adolescents. Neuroscience letters, 441(2), 219-223.

Byun, K., Hyodo, K., Suwabe, K., Ochi, G., Sakairi, Y., Kato, M., ... \& Soya, H. (2014). Positive effect of acute mild exercise on executive function via arousal-related prefrontal activations: an fNIRS study. Neuroimage, 98, 336-345. 
Cahill, L., \& Alkire, M. T. (2003). Epinephrine enhancement of human memory consolidation: interaction with arousal at encoding. Neurobiology of learning and memory, 79(2), 194- 198.

Cahill, L., Gorski, L., \& Le, K. (2003). Enhanced human memory consolidation with postlearning stress: interaction with the degree of arousal at encoding. Learning \& memory, 10(4), 270-274.

Calvert, H., Hwang, J., Kim, K., Harrison, M., Brothers, R., Glowacki, E., ... \& Castelli, D. (2014). The impact of acute exercise on brain-derived neurotropic factor (BDNF) and cognitive performance (LB694). The FASEB Journal, 28(1 Supplement), LB694.

Castelli, D. M., Hillman, C. H., Buck, S. M., \& Erwin, H. E. (2007). Physical fitness and academic achievement in third-and fifth-grade students. Journal of Sport and Exercise Psychology, 29(2), 239.

Centers for Disease Control and Prevention (CDC). (2004). BMI-Body Mass Index: BMI Calculator. Accessed on April, 2, 2005.

Centers for Disease Control and Prevention, \& Centers for Disease Control and Prevention. (2010). The association between school based physical activity, including physical education, and academic performance. Atlanta, GA: US Department of Health and Human Services, 9.

Chen, A. G., Yan, J., Yin, H. C., Pan, C. Y., \& Chang, Y. K. (2014). Effects of acute aerobic exercise on multiple aspects of executive function in preadolescent children. Psychology of Sport and Exercise, 15(6), 627-636. 
Coe, D. P., Pivarnik, J. M., Womack, C. J., Reeves, M. J., \& Malina, R. M. (2006). Effect of physical education and activity levels on academic achievement in children. Medicine and science in sports and exercise, 38(8), 1515.

Coles, K., \& Tomporowski, P. D. (2008). Effects of acute exercise on executive processing, short-term and long-term memory. Journal of sports sciences, 26(3), 333-344.

Constantini, N. W., \& Hackney, A. C. (Eds.). (2013). Endocrinology of physical activity and sport. New York: Humana Press.

Del Corral, P., Mahon, A. D., Duncan, G. E., Howe, C. A., \& Craig, B. W. (1994). The effect of exercise on serum and salivary cortisol in male children. Medicine and Science in Sports and Exercise, 26(11), 1297-1301.

Diamond, A. (2000). Close interrelation of motor development and cognitive development and of the cerebellum and prefrontal cortex. Child development, 71(1), 44-56.

Diamond, A. (2013). Executive functions. Annual review of psychology, 64, 135-168.

Donchin, E. (1981). Surprise!... surprise?. Psychophysiology, 18(5), 493-513.

Duncko, R., Johnson, L., Merikangas, K., \& Grillon, C. (2009). Working memory performance after acute exposure to the cold pressor stress in healthy volunteers. Neurobiology of learning and memory, 91(4), 377-381.

Dustman, R. E., Emmerson, R. Y., Ruhling, R. O., Shearer, D. E., Steinhaus, L. A., Johnson, S. C., ... \& Shigeoka, J. W. (1990). Age and fitness effects on EEG, ERPs, visual sensitivity, and cognition. Neurobiology of aging, 11(3), 193-200.

Ellemberg, D., \& St-Louis-Deschênes, M. (2010). The effect of acute physical exercise on cognitive function during development. Psychology of Sport and Exercise, 11(2), 122-126. 
Engel, F., Härtel, S., Strahler, J., Wagner, M. O., Bös, K., \& Sperlich, B. (2014). Hormonal, metabolic, and cardiorespiratory responses of young and adult athletes to a single session of high-intensity cycle exercise. Pediatric exercise science, 26(4), 485494.

Etnier, J. L., Nowell, P. M., Landers, D. M., \& Sibley, B. A. (2006). A meta-regression to examine the relationship between aerobic fitness and cognitive performance. Brain research reviews, 52(1), 119-130.

Etnier, J. L., Salazar, W., Landers, D. M., Petruzzello, S. J., Han, M., \& Nowell, P. (1997). The Influence of Physical Fitness and Exercise Upon Cognitive Functioning: A Meta-Analysis. Journal of sport \& exercise psychology, 19(3), 249-277.

Etnier, J. L., Salazar, W., Landers, D. M., Petruzzello, S. J., Han, M., \& Nowell, P. (1997). The influence of physical fitness and exercise upon cognitive functioning: a metaanalysis. Journal of sport and Exercise Psychology, 19(3), 249-277.

Giles, G. E., Mahoney, C. R., Brunyé, T. T., Taylor, H. A., \& Kanarek, R. B. (2014). Stress effects on mood, HPA axis, and autonomic response: comparison of three psychosocial stress paradigms. PloS one, $9(12)$, e113618.

Goekint, M., Heyman, E., Roelands, B., Njemini, R., Bautmans, I., Mets, T., \& Meeusen, R. (2008). No influence of noradrenaline manipulation on acute exercise-induced increase of brain-derived neurotrophic factor. Med Sci Sports Exerc, 40(11), 1990-6.

Hackney, A. C., McMurray, R. G., Judelson, D. A., \& Harrell, J. S. (2003). Relationship between caloric intake, body composition, and physical activity to leptin, thyroid 
hormones, and cortisol in adolescents. The Japanese journal of physiology, 53(6), $475-479$

Hackney, A. C., McMurray, R. G., Judelson, D. A., \& Harrell, J. S. (2003). Relationship between caloric intake, body composition, and physical activity to leptin, thyroid hormones, and cortisol in adolescents. The Japanese journal of physiology, 53(6), 475-479.

Henckens, M. J., van Wingen, G. A., Joëls, M., \& Fernández, G. (2011). Time-dependent corticosteroid modulation of prefrontal working memory processing. Proceedings of the National Academy of Sciences, 108(14), 5801-5806.

Hill, E. E., Zack, E., Battaglini, C., Viru, M., Viru, A., \& Hackney, A. C. (2008). Exercise and circulating cortisol levels: the intensity threshold effect. Journal of endocrinological investigation, 31(7), 587-591.

Hillman, C. H., Belopolsky, A. V., Snook, E. M., Kramer, A. F., \& McAuley, E. (2004). Physical activity and executive control: implications for increased cognitive health during older adulthood. Research quarterly for exercise and sport, 75(2), 176-185.

Hillman, C. H., Castelli, D. M., \& Buck, S. M. (2005). Aerobic fitness and neurocognitive function in healthy preadolescent children. Medicine and science in sports and exercise, 37(11), 1967.

Hillman, C. H., Pontifex, M. B., Raine, L. B., Castelli, D. M., Hall, E. E., \& Kramer, A. F. (2009). The effect of acute treadmill walking on cognitive control and academic achievement in preadolescent children. Neuroscience, 159(3), 1044-1054. 
Horswill, C. A., Zipf, W. B., Kien, C. L., \& Kahle, E. B. (1997). Insulin's contribution to growth in children and the potential for exercise to mediate insulin's action. Pediatric Exercise Science, 9(1), 18-32.

Hötting, K., Schickert, N., Kaiser, J., Röder, B., \& Schmidt-Kassow M. (2016). The effects of acute physical exercise on memory, peripheral BDNF, and cortisol in young adults. Neural Plasticity, 2016

Ide, K., \& Secher, N. H. (2000). Cerebral blood flow and metabolism during exercise. Progress in neurobiology, 61(4), 397-414.

ISURUGI, K., FUKUTANI, K., TAKAYASU, H., WAKABAYASHI, K., \& TAMAOKI, B. I. (1974). Age-Related Changes in Serum Luteinizing Hormone (LH) and Follicle-Stimulating Hormone (FSH) Levels in Normal Men 1. The Journal of Clinical Endocrinology \& Metabolism, 39(5), 955-957.

Joëls, M., Fernandez, G., \& Roozendaal, B. (2011). Stress and emotional memory: a matter of timing. Trends in cognitive sciences, 15(6), 280-288.

Kamijo, K., Nishihira, Y., Hatta, A., Kaneda, T., Wasaka, T., Kida, T., \& Kuroiwa, K. (2004). Differential influences of exercise intensity on information processing in the central nervous system. European journal of applied physiology, 92(3), 305-311.

Kamijo, K., Nishihira, Y., Higashiura, T., Hatta, A., Kaneda, T., Kim, S., ... \& Kim, B. (2006). Influence of exercise intensity on cognitive processing and arousal level in the central nervous system. Advances in exercise and sports physiology, 12(1), 1.

Kamijo, K., Hayashi, Y., Sakai, T., Yahiro, T., Tanaka, K., \& Nishihira, Y. (2009). Acute effects of aerobic exercise on cognitive function in older adults. The Journals of 
Gerontology Series B: Psychological Sciences and Social Sciences, 64(3), 356363.

Kilian, Y., Engel, F., Wahl, P., Achtzehn, S., Sperlich, B., \& Mester, J. (2016). Markers of biological stress in response to a single session of high-intensity interval training and high-volume training in young athletes. European Journal of Applied Physiology, 110.

Kirschbaum, C., \& Hellhammer, D. H. (1994). Salivary cortisol in psychoneuroendocrine research: recent developments and applications. Psychoneuroendocrinology, 19(4), 313-333.

Kubesch, S., Walk, L., Spitzer, M., Kammer, T., Lainburg, A., Heim, R., \& Hille, K. (2009). A 30-Minute Physical Education Program Improves Students' Executive Attention. Mind, Brain, and Education, 3(4), 235-242.

Kuhlmann, S., \& Wolf, O. T. (2006). Arousal and cortisol interact in modulating memory consolidation in healthy young men. Behavioral neuroscience, 120(1), 217.

Labelle, V., Bosquet, L., Mekary, S., \& Bherer, L. (2013). Decline in executive control during acute bouts of exercise as a function of exercise intensity and fitness level. Brain and cognition, 81(1), 10-17.

Lambourne, K., \& Tomporowski, P. (2010). The effect of exercise-induced arousal on cognitive task performance: a meta-regression analysis. Brain research, 1341, 1224.

Lambrick, D., Stoner, L., Grigg, R., \& Faulkner, J. (2016). Effects of continuous and intermittent exercise on executive function in children aged 8-10 years. Psychophysiology, 53(9), 1335-1342. 
Lang, P. J., Bradley, M. M., \& Cuthbert, B. N. (1997). International affective picture system (IAPS): Technical manual and affective ratings. NIMH Center for the Study of Emotion and Attention, 39-58.

Li, G., Cherrier, M. M., Tsuang, D. W., Petrie, E. C., Colasurdo, E. A., Craft, S., ... \& Wilkinson, C. W. (2006). Salivary cortisol and memory function in human aging. Neurobiology of aging, 27(11), 1705-1714.

Luethi, M., Meier, B., \& Sandi, C. (2009). Stress effects on working memory, explicit memory, and implicit memory for neutral and emotional stimuli in healthy men. Frontiers in behavioral neuroscience, 2, 5.

Lupien, S. J., \& Lepage, M. (2001). Stress, memory, and the hippocampus: can't live with it, can't live without it. Behavioural brain research, 127(1), 137-158.

Lupien, S. J., de Leon, M., De Santi, S., Convit, A., Tarshish, C., Nair, N. P. V., ... \& Meaney, M. J. (1998). Cortisol levels during human aging predict hippocampal atrophy and memory deficits. Nature neuroscience, 1(1), 69-73.

Lupien, S. J., Fiocco, A., Wan, N., Maheu, F., Lord, C., Schramek, T., \& Tu, M. T. (2005). Stress hormones and human memory function across the lifespan. Psychoneuroendocrinology, 30(3), 225-242.

Martin, F., Delpont, E., Suisse, G., Richelme, C., \& Dolisi, C. (1993). Long latency eventrelated potentials (P300) in gifted children. Brain and development, 15(3), 173177.

McAuley, E., Kramer, A. F., \& Colcombe, S. J. (2004). Cardiovascular fitness and neurocognitive function in older adults: a brief review. Brain, behavior, and immunity, 18(3), 214-220. 
McEwen, B. S. (2004). Protection and damage from acute and chronic stress: allostasis and allostatic overload and relevance to the pathophysiology of psychiatric disorders. Annals of the New York Academy of Sciences, 1032(1), 1-7.

McMorris, T. (2009). Exercise and cognitive function: a neuroendocrinological explanation. Exercise and cognitive function, 41-68.

McMorris, T., \& Hale, B. J. (2012). Differential effects of differing intensities of acute exercise on speed and accuracy of cognition: a meta-analytical investigation. Brain and cognition, 80(3), 338-351.

McMorris, T., Collard, K., Corbett, J., Dicks, M., \& Swain, J. P. (2008). A test of the catecholamines hypothesis for an acute exercise-cognition interaction. Pharmacology Biochemistry and Behavior, 89(1), 106-115.

Miller, E. K., \& Cohen, J. D. (2001). An integrative theory of prefrontal cortex function. Annual review of neuroscience, 24(1), 167-202.

Moriguchi, Y., \& Hiraki, K. (2014). Behavioral and neural differences during two versions of cognitive shifting tasks in young children and adults. Developmental psychobiology, 56(4), 761-769.

Oei, N. Y. L., Everaerd, W. T. A. M., Elzinga, B. M., Van Well, S., \& Bermond, B. (2006). Psychosocial stress impairs working memory at high loads: an association with cortisol levels and memory retrieval. Stress, 9(3), 133-141.

ORENTREICH, N., BRIND, J. L., RIZER, R. L., \& VOGELMAN, J. H. (1984). Age changes and sex differences in serum dehydroepiandrosterone sulfate concentrations throughout adulthood. The Journal of Clinical Endocrinology \& Metabolism, 59(3), 551-555. 
Payne, J. D., Jackson, E. D., Hoscheidt, S., Ryan, L., Jacobs, W. J., \& Nadel, L. (2007). Stress administered prior to encoding impairs neutral but enhances emotional long-term episodic memories. Learning \& Memory, 14(12), 861-868.

Pesce, C., \& Audiffren, M. (2011). Does acute exercise switch off switch costs? A study with younger and older athletes. Journal of Sport and Exercise Psychology, 33(5), 609626.

Pesce, C., Crova, C., Cereatti, L., Casella, R., \& Bellucci, M. (2009). Physical activity and mental performance in preadolescents: Effects of acute exercise on free-recall memory. Mental Health and Physical Activity, 2(1), 16-22.

Plessow, F., Fischer, R., Kirschbaum, C., \& Goschke, T. (2011). Inflexibly focused under stress: acute psychosocial stress increases shielding of action goals at the expense of reduced cognitive flexibility with increasing time lag to the stressor. Journal of cognitive neuroscience, 23(11), 3218-3227.

Polich, J., \& Lardon, M. T. (1997). P300 and long-term physical exercise. Electroencephalography and Clinical Neurophysiology, 103(4), 493498.

Polich, J., Ladish, C., \& Bloom, F. E. (1990). P300 assessment of early Alzheimer's disease. Electroencephalography and Clinical Neurophysiology/Evoked Potentials Section, 77(3), 179-189.

Pontifex, M., Hillman, C., Fernhall, B. O., Thompson, K., \& Valentini, T. (2009). The effect of acute aerobic and resistance exercise on working memory. MedicineScience in Sports + Exercise, 41(4), 927.18. 
Purifoy, F. E., Koopmans, L. H., \& Tatum, R. W. (1980). Steroid hormones and aging: free testosterone, testosterone and androstenedione in normal females aged 20-87 years. Human biology, 181-191.

Querido, J. S., \& Sheel, A. W. (2007). Regulation of cerebral blood flow during exercise. Sports Medicine, 37(9), 765-782.

Richter-Levin, G., \& Akirav, I. (2003). Emotional tagging of memory formation - in the search for neural mechanisms. Brain Research Reviews, 43(3), 247-256.

Roberts, A. C., Robbins, T. W., \& Weiskrantz, L. E. (1998). The prefrontal cortex: Executive and cognitive functions. Oxford University Press.

Roozendaal, B. (2000). Glucocorticoids and the regulation of memory consolidation. Psychoneuroendocrinology, 25(3), 213-238.

Roozendaal, B., McEwen, B. S., \& Chattarji, S. (2009). Stress, memory and the amygdala. Nature Reviews Neuroscience, 10(6), 423-433.

Schoofs, D., Pabst, S., Brand, M., \& Wolf, O. T. (2013). Working memory is differentially affected by stress in men and women. Behavioural brain research, 241, 144-153.

Schoofs, D., Preuß, D., \& Wolf, O. T. (2008). Psychosocial stress induces working memory impairments in an n-back paradigm. Psychoneuroendocrinology, 33(5), 643-653.

Schoofs, D., Wolf, O. T., \& Smeets, T. (2009). Cold pressor stress impairs performance on working memory tasks requiring executive functions in healthy young men. Behavioral neuroscience, 123(5), 1066.

Schwabe, L., Höffken, O., Tegenthoff, M., \& Wolf, O. T. (2013). Stress-induced enhancement of response inhibition depends on mineralocorticoid receptor activation. Psychoneuroendocrinology, 38(10), 2319-2326. 
Shansky, R. M., \& Lipps, J. (2013). Stress-induced cognitive dysfunction: hormoneneurotransmitter interactions in the prefrontal cortex. Frontiers in human neuroscience, 7.

Shields, G. S., Bonner, J. C., \& Moons, W. G. (2015). Does cortisol influence core executive functions? A meta-analysis of acute cortisol administration effects on working memory, inhibition, and set-shifting. Psychoneuroendocrinology, 58, 91103.

Shields, G. S., Sazma, M. A., \& Yonelinas, A. P. (2016). The effects of acute stress on core executive functions: A meta-analysis and comparison with cortisol. Neuroscience \& Biobehavioral Reviews, 68, 651-668.

Sibley, B. A., \& Etnier, J. L. (2003). The relationship between physical activity and cognition in children: a meta-analysis. Pediatric exercise science, 15(3), 243-256.

Smeets, T., Otgaar, H., Candel, I., \& Wolf, O. T. (2008). True or false? Memory is differentially affected by stress-induced cortisol elevations and sympathetic activity at consolidation and retrieval. Psychoneuroendocrinology, 33(10), 13781386.

Soga, K., Shishido, T., \& Nagatomi, R. (2015). Executive function during and after acute moderate aerobic exercise in adolescents. Psychology of Sport and Exercise, 16, 7-17.

Stauder, J. E., van der Molen, M. W., \& Molenaar, P. C. (2003). Age, intelligence, and eventrelated brain potentials during late childhood: A longitudinal study. Intelligence, 31(3), 257-274. 
Taverniers, J., Van Ruysseveldt, J., Smeets, T., \& von Grumbkow, J. (2010). Highintensity stress elicits robust cortisol increases, and impairs working memory and visuo- spatial declarative memory in Special Forces candidates: A field experiment. Stress, 13(4), 324-334.

Tomporowski, P. D. (2003). Effects of acute bouts of exercise on cognition. Acta psychologica, 112(3), 297-324.

Tsai, C. L., Wang, C. H., Pan, C. Y., Chen, F. C., Huang, T. H., \& Chou, F. Y. (2014). Executive function and endocrinological responses to acute resistance exercise. Frontiers in behavioral neuroscience, 8, 262.

Tsukamoto, H., Suga, T., Takenaka, S., Tanaka, D., Takeuchi, T., Hamaoka, T., ... \& Hashimoto, T. (2016). Greater impact of acute high-intensity interval exercise on post-exercise executive function compared to moderate-intensity continuous exercise. Physiology \& behavior, 155, 224-230.

Tulsky, D. S., Carlozzi, N., Chiaravalloti, N. D., Beaumont, J. L., Kisala, P. A., Mungas, D., ... \& Gershon, R. (2014). NIH Toolbox Cognition Battery (NIHTB-CB): List sorting test to measure working memory. Journal of the International Neuropsychological Society, 20(6), 599-610.

Ulrich-Lai, Y. M., \& Herman, J. P. (2009). Neural regulation of endocrine and autonomic stress responses. Nature Reviews Neuroscience, 10(6), 397-409.

van Peer, J. M., Roelofs, K., \& Spinhoven, P. (2008). Cortisol administration enhances the coupling of midfrontal delta and beta oscillations. International Journal of Psychophysiology, 67(2), 144-150. 
Verburgh, L., Königs, M., Scherder, E. J., \& Oosterlaan, J. (2013). Physical exercise and executive functions in preadolescent children, adolescents and young adults: a meta-analysis. British journal of sports medicine, bjsports-2012.

Wang, H. X., Jin, Y., Hendrie, H. C., Liang, C., Yang, L., Cheng, Y., ... \& Li, P. (2013). Late life leisure activities and risk of cognitive decline. The Journals of Gerontology Series A: Biological Sciences and Medical Sciences, 68(2), 205-213.

Wilson, A. N., Olds, T., Lushington, K., Petkov, J., \& Dollman, J. (2016). The impact of 10minute activity breaks outside the classroom on male students' on-task behaviour and sustained attention: a randomised crossover design. Acta paediatrica, 105(4), e181-e188.

Wilson, A. N., Olds, T., Lushington, K., Petkov, J., \& Dollman, J. (2016). The impact of 10minute activity breaks outside the classroom on male students' on-task behaviour and sustained attention: a randomised crossover design. Acta paediatrica, 105(4), e181-e188. 


\title{
APPENDICES
}

\author{
Appendix A \\ Informed Consent Form
}

\section{California Polytechnic State University
Informed Consent to Participate in a Research Project Examining the Effects of Exercise-Induced Hormones on Memory and Attention

Investigators: A research project focused on the effects of moderate-intensity aerobic exercise on hormonal and enzyme levels, and the effects they have on tests of memory and attention. This project is being conducted by Cory Greever Ph.D.

(greever@calpoly.edu) and Kory Bettencourt (kmbetten@calpoly.edu) in the Department of Kinesiology and Psychology and Child Development at Cal Poly, San Luis Obispo.

Note: You are being asked to give consent to allow the child you are caring for to participate in a research study. Participation is voluntary. Please feel free to ask questions at any time if there is something you do not understand. You may omit to any questions you prefer not to answer about the child you are representing. The child may discontinue participation at any time without penalty or loss of benefits.

Purpose: The purpose of this study is to examine the effects of exercise-induced hormone release on two aspects of cognitive functioning: working memory and attention. A total of 30 healthy children between $3^{\text {rd }}$ and $5^{\text {th }}$ grade will be recruited for the study. There will be two visits to the Kinesiology Department and each visit will last between 12 hours. During the first visit, participants will be randomized into 1 of 2 conditions, an exercise or resting condition. The exercise condition consists of 30 minutes of moderate intensity exercise on a treadmill. The resting condition will consist of 30 minutes of quietly sitting while watching an age appropriate television show. After a one-week wash out period, the participant will return to the lab for their $2^{\text {nd }}$ visit. This visit will have the participant flip-flop condition based on their $1^{\text {st }}$ visit (rest to exercise, exercise to rest). Trained research assistants will collect salivary cortisol sample immediately before and immediately after the 30 minutes of rest and exercise. Two separate cognitive tests will be administered immediately after the both conditions.

Risks and Discomforts: There are minimal risks involved with this study. Trained individuals will conduct all laboratory procedures with the child's well-being being the first priority. All procedures will be explained to you, and the child until you are comfortable with the proposed study.

Exercise: During any type of exercise, there are slight health risks along with the possibility of fatigue and muscle soreness. Health risks are minor in people with no prior history of cardiovascular, respiratory, and musculoskeletal disease or injury. Other potential discomforts include stomach pain, dizziness, and shortness of breath. There is also an extremely rare chance of death. 
Prior to the study: All children should report to the Cal Poly Human Performance lab between the hours of 3 and $6 \mathrm{pm}$. Children should be fasted for 2 hours prior to each visit and should refrain from any physical activity, beverages (Gatorade, soda, etc.), and brushing their teeth 2 hours prior to each visit. Children can drink water up to 30 minutes before each visit.

Salivary Hormone Samples: A SalivaBio Oral Swab (SOS) will be collected at various times during both visits. This saliva collection is intended to sample hormones such as cortisol and testosterone, and the enzyme salivary alpha-amylase. The collection will be conducted immediately before and immediately after the rest/or exercise condition. During the collection, participants place a small swab under their tongue and salivate on it for 1-2 minutes. After the saliva is collected, it will be labeled and stored confidentially. We will demonstrate to yourself and the child the saliva collection methods. If you or the child do not feel comfortable with any part of the process, the samples will not be collected to no penalty or loss of benefits to you or the child.

Exercise: This study will require participants to exercise for 30-minutes at moderate intensity for 1 of the 2 visits. The exercise will be conducted on a treadmill, with trained research assistants making sure the participant is as safe and comfortable as possible. We will have the participants exercise at $70 \%$ of their heart rate max determined by a heart rate maximum equation (220-age). Participants will be informed of the protocol and will be given the opportunity to ask any questions. Heart rate and Ratings of Perceived Exertion (RPE) will be monitored throughout the exercise. If at any time during the exercise the participant feels any pain or discomfort, they can stop the treadmill and the exercise without loss of benefits. If you have any questions regarding the exercise protocol, you can ask any of the primary researchers.

Accelerometers: After the first visit to Human Performance Lab, participants will be asked to wear an Actigraph accelerometer around their left wrist. The accelerometer is a way to measure physical activity levels during the one-week washout period. Accelerometers should be worn at all times except when bathing, showering, or swimming. If you have any questions regarding the accelerometer, or feel uncomfortable with the child wearing it, you can discuss these issues with any of the primary researchers.

Benefits: There are no direct benefits to you or the child in the participation of this study. The data are collected purely for the purposes of research and do not have a clinical or diagnostic value. However, these data may further the understanding of whether increased cortisol after exercise can increase the cognitive functioning among children. After completion of the study, you will be offered the opportunity to view the results of the study.

Confidentiality: All records and data collected during this study will be treated as confidential. Your name, and the participating child's name will be kept confidential. 
Information stored on our laptops, computers, or iPad will be password protected. Only research staff and the principle investigator will have access to the data.

Costs/Payments: There are no costs to you or the child for participating in this study. Each participant will be rewarded with a gift for their participation in this study.

Questions: If you have any questions regarding this study, or would like to be informed of the results when the study is completed, please feel free to contact Dr. Cory Greever (greever@,calpoly.edu; 805-756-2103) or Kory Bettencourt (kmbetten@calpoly.edu; 209-648-3042). If you have any concerns regarding the manner in which the study is being conducted, you may contact Dr. Michael Black, Chair of the Cal Poly human Subjects Committee at (805) 756-2894, mblack@,calpoly.edu, or Dr. Dean Wendt, Dean of Research at (805) 756-1508, dwendt@calpoly.edu.

If you agree to allow the child you are representing to participate in the research project described, please indicate your agreement by signing below. Please keep one copy of this form for reference, and thank you for your participation in this research.

Signature of Guardian

Date

Signature of Researcher

Date 
Appendix B

Informed Assent Form

\section{Effects of Exercise-Induced Hormones on Working Memory and Attention}

\section{Investigators: Dr. Cory Greever, Todd Hagobian, Dr. Kelly Bennion, and Kory Bettencourt}

We are doing a research project about whether exercise makes people's brains work better. We suspect that exercise causes changes in the body that might help kids remember information and pay attention. If you decide that you want to be a part of this study, you will be asked to come to Cal Poly Human Performance Laboratory twice for testing and measurements.

There are some things about this study you should know. These are:

Visit 1

- Height

- Weight

- Saliva collection - we will ask you to put a small cotton $\operatorname{swab}(\rightarrow)$ in your mount for 1-2 minutes (2 times)

- Sitting quietly for 30-minutes while watching t.v. OR exercising for 30 minutes on a treadmill

- We will randomize whether you exercise or watch t.v., meaning we all will not know what you are doing until that day.

- Cognitive Tests - we will do fun brain games before and after you sit to see if there are any changes.

- We will also put an accelerometer on your wrist before you leave. This will measure how your body moves.

Visit 2

- Saliva sample - we will ask you to put a small cotton swab in your mouth for 1-2 minutes. (2 times)

- Exercise on a treadmill for 30-minutes OR watching t.v for 30 minutes (depending on what you did during visit 1). If you exercise during the $1^{\text {st }}$ visit you will rest this visit, and if you rest the $1^{\text {st }}$ visit, you will exercise this visit.

- Cognitive Tests - we will do fun brain games before and after you exercise, to see if there are any changes.

What are the benefits?

Not everyone who takes part in this study will benefit. A benefit means something good happens to you. You will get a chance to see how fit your heart and lungs are, which is very important for health. For being in this study, you will get a $\$ 20$ gift card each visit to the Cal Poly lab.

Can anything bad happen to me? 
While it is not likely, there are some bad things that could happen during the treadmill exercise including:

- You may feel a little sick to your stomach

- You may get dizzy

- You may get out of breath

- Your muscles may get sore for 2-4 days.

- You can hurt some of your muscles or bones.

During this exercise, if you feel strange or that something is wrong, you can end the exercise. We want you to be comfortable, so you can tell us if something does not feel right or is hurting you.

\section{Discomforts}

When we collect saliva, we will have you put a swab under your tongue for 1-2 minutes. This may feel uncomfortable to you. If you feel that you do not want to do this, you can say so, or you can stop the saliva collection at any time.

Who can I talk to about the study?

You can talk to your primary care-giver, Dr. Cory Greever, or Kory Bettencourt about the study. We will be here to answer any questions you have. You can ask any question you want at any time and we will answer them for you.

What if I do not want to do this?

If you do not want to do this study, you tell your primary caregiver, Dr. Greever, or Kory Bettencourt at any time. We will not be mad at you at all. It is completely up to you to decide if you want to be in this study or not. At any time, you can tell one of us that you do not feel comfortable, or do not want to do something, and we will allow you to stop.

When we are finished with this study we will write a report about what we learned. Your name will not be in the report.

If you decide to be in this study, please sign your name.

I, , want to be in this research study.

Sign here

Date 
Appendix C

Health Screener

\section{ID \# \\ Test Subject Screener \\ THE EFFECT OF EXERCISE-INDUCED CORTISOL ON EXECUTIVE FUNCTIONING IN PREADOLSCENTS}

Instructions: This form will be used as a guide when talking on the phone or face-toface with parents and or guardians of the potential study participant. Before asking any questions, inform the guardian the study and what participation entails. Identify the participant by ID\# NOT name.

Introduce self

$\rightarrow$ "Hello, my name is from the Department of Kinesiology at Cal Poly, San Luis Obispo."

Tell the person why you are calling and the purpose of the research study:

$\rightarrow$ "We are working on research about the effects of exercised induced cortisol release on cognitive functioning in preadolescents."

Request permission to ask questions to see if the individual' child qualifies for the research study.

$\rightarrow$ "Would it be ok if I asked you a few questions regarding your child's eligibility for our study?"

If Person says "no", thank the person for his/her time and politely end the call. If the person says "yes" inform them about the use of private health information that will be collected regarding their child during the phone call.

$\rightarrow$ "We will be collecting information regarding your child during this phone call. You taking part in this phone call is completely voluntary."

$\rightarrow$ "Your child's information will only be seen by researchers at Cal Poly University. We keep information completely confidential."

Begin the screener form.

If the guardian answers "yes" to any of the pre-existing conditions, child is left handed, or speaks any language other than English as their first language, they are deemed ineligible to participate in the study. Politely explain this to the guardian and thank them for their time.

$\rightarrow$ "Unfortunately, due to (list reason here), your child is ineligible to participate in this study. We thank you for your time. If you have any other questions, feel free to contact us."

If the child meets screening eligibility, describe the study procedures, including the number of visits and how long the person will be in the study.

Describe the potential risks.

Describe the benefits

After explanation of the study, ask the following:

"Do you have any questions?"

"Do you think your child would like to take part in this research?" 
Provide the person with contact name and number for any further questions about the study.

\begin{tabular}{|c|c|}
\hline Gender of the Participant & Male/Female \\
\hline \multicolumn{2}{|l|}{ Date of Birth } \\
\hline \multicolumn{2}{|l|}{ Highest Education Level Completed by Participant } \\
\hline Handedness & Left/Right \\
\hline \multicolumn{2}{|l|}{ Race } \\
\hline \multicolumn{2}{|l|}{ Ethnicity } \\
\hline English as a fist language & Yes/No \\
\hline Does participant have any pre-existing heart conditions? & Yes/No \\
\hline Does participant have any pre-existing lung conditions? & Yes/No \\
\hline $\begin{array}{l}\text { Does participant have any pre-existing metabolic } \\
\text { conditions? }\end{array}$ & Yes/No \\
\hline Does participant have any emotional or mental disorders? & Yes/No \\
\hline $\begin{array}{l}\text { Has your child reached, or shown signs of reaching } \\
\text { puberty? }\end{array}$ & Yes/No \\
\hline $\begin{array}{l}\text { Does the participant have any major-medical conditions } \\
\text { that prohibit physical activity? }\end{array}$ & Yes/No \\
\hline Does child participate in free and reduced lunch? & Yes/No \\
\hline \multicolumn{2}{|l|}{$\begin{array}{l}\text { What is the highest level of education obtained by the } \\
\text { mother? }\end{array}$} \\
\hline $\begin{array}{l}\text { What is the highest level of education obtained by the } \\
\text { father? }\end{array}$ & \\
\hline
\end{tabular}


Number of parents that work full time? 


\author{
Appendix D \\ Child Stress Disorder Checklist (CSDC)
}

\title{
CHILD STRESS DISORDERS CHECKLIST (CSDC)
}

(v. 4.0- 11/01)

Glenn N. Saxe, M.D.

National Child Traumatic Stress Network \&

Department of Child and Adolescent Psychiatry,

Boston University School of Medicine

glenn.saxe@bmc.org

Child's Name (or ID \#):

Age:

Sex: $\mathbf{M} \quad \mathbf{F}$

Person Completing Questionnaire:

Date

Relationship to Child:

Has your child experienced or witnessed an event that caused, or threatened to cause, serious harm to him or herself or to someone else? Please check any and all events (and age(s) of your child at the time of the event or events) below-

1) Car Accident Age(s)

2) Other Accident Age(s)

3) Fire Age(s)

4) Storm Age(s)

5) Physical Illness
6) Physical Assault
7) Sexual Assault
8) Any Other Even Age(s) Age(s) Age(s)

Please provide any details about this (or these) events in the box below. For exampleWhere did the event occur? Who was with your child? Who hurt your child? How often did this happen? How long did it last? How badly was your child hurt? Did he or she require medical care? 
Directions: Below is a list of feelings or behaviors that children sometimes have immediately after a frightening event (or after he or she regained consciousness from such an event). For each item that describes your child immediately after the event, please circle 2 if the item is VERY TRUE of your child. Circle 1 if the item is SOMEWHAT TRUE of your child. If the item is NOT TRUE of your child, circle 0 . Please answer all items as well as you can even if some do not seem to apply to your child. For children who have experienced more than one event, choose the event that was most distressing to him or her.

\section{$0=$ Not True (as far as you know) $1=$ Somewhat True $2=$ Very True}

012 1) Child felt terrified (extreme anxiety or fear).

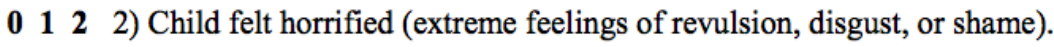

$\begin{array}{lll}0 & 1 & 2\end{array}$ ) Child felt helpless.

012 4) Child's behavior became agitated. For example, his or her behavior became hyperactive, impulsive, or difficult to control.

$\begin{array}{lll}0 & 1 & 2\end{array}$ ) Child's behavior became disorganized. For example, his or her behavior became very different than is usual, his or her behavior did not make sense. 
Directions: Below is a list of behaviors that describe children. For each item that describes your child NOW or WITHIN THE PAST MONTH, please circle 2 if the item is VERY TRUE or OFTEN TRUE of your child. Circle $\mathbf{1}$ if the item is SOMEWHAT or SOMETIMES TRUE of your child. If the item is NOT TRUE of your child, circle 0. Please answer all items as well as you can even if some do not seem to apply to your child. The term "event" refers to the most stressful experience that you have described above.

\section{$0=$ Not True (as far as you know) $1=$ Somewhat or Sometimes True $2=$ Very True or Often True}

$\left.\begin{array}{lll}0 & 2 & 1\end{array}\right)$ Child reports uncomfortable memories of the event.

012 2) Child startles easily. For example, he or she jumps when hears sudden or loud noises.

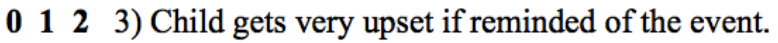

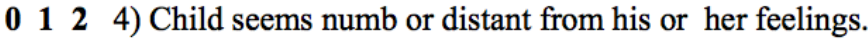

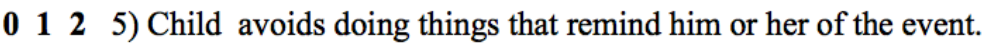

012 6) Child seems irritable or angry.

$\begin{array}{lll}0 & 1 & 2\end{array}$ 7) Child has difficulty remembering details about the event.

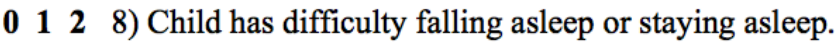

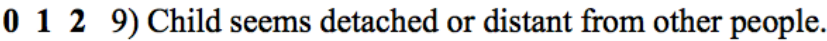

012 10) Child has difficulty getting along with friends, schoolmates or teachers.

012 11) Child does things that he or she outgrew. For example, thumb sucking, bedwetting, nail biting, or requests to sleep with parents.

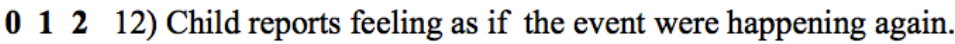

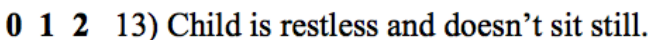

012 14) Child avoids places that remind him or her of the event. 
Directions: Below is a list of behaviors that describe children. For each item that describes your child NOW or WITHIN THE PAST MONTH, please circle 2 if the item is VERY TRUE or OFTEN TRUE of your child. Circle 1 if the item is SOMEWHAT or SOMETIMES TRUE of your child. If the item is NOT TRUE of your child, circle 0 . Please answer all items as well as you can even if some do not seem to apply to your child. The term "event" refers to the most stressful experience that you have described above.

0 = Not True (as far as you know) 1 = Somewhat or Sometimes True $2=$ Very True or Often True

012 1) Child reports uncomfortable memories of the event.

012 2) Child startles easily. For example, he or she jumps when hears sudden or loud noises.

$\left.\begin{array}{llll}0 & 1 & 2 & 3\end{array}\right)$ Child gets very upset if reminded of the event.

012 4) Child seems numb or distant from his or her feelings.

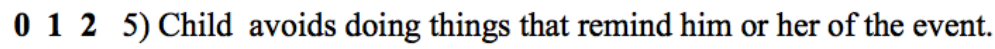

012 6) Child seems irritable or angry.

$\begin{array}{lll}0 & 1 & 2\end{array}$ ) Child has difficulty remembering details about the event.

$\left.\begin{array}{lll}0 & 2 & 2\end{array}\right)$ Child has difficulty falling asleep or staying asleep.

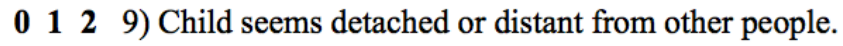

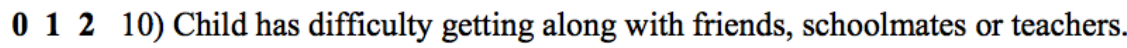

012 11) Child does things that he or she outgrew. For example, thumb sucking, bedwetting, nail biting, or requests to sleep with parents.

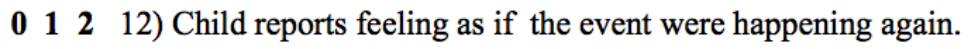

$\left.\begin{array}{lll}0 & 2 & 13)\end{array}\right)$ Child is restless and doesn't sit still.

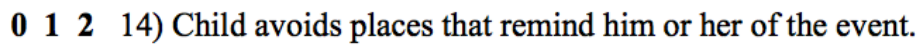




\section{SCORING THE CHILD STRESS DISORDERS CHECKLIST}

The Child Stress Disorders Checklist (CSDC) assesses a child's post-traumatic symptoms based on observer report. Observers respond to an inventory of symptoms by indicating $\mathbf{0}$ (Not True), $\mathbf{1}$ (Somewhat or Sometimes True), or $\mathbf{2}$ (Very True) based on their observations of their child. Scores are calculated by adding the responses within a variety of dimensions of post traumatic symptoms.

The first page of the CSDC gathers descriptive information about the traumatic event or events. This page is not meant to be scored but yields information about the type(s) of traumatic events that the child may have experienced, his or her age(s) at the time of the event, as well as a qualitative description of the circumstances of the event.

The second to fourth pages of this instrument gather quantitative information about a child's post traumatic symptoms.

The first five items of the CSDC asks about a child's immediate responses to the event. The Immediate Response Score is calculated by adding the scores $(0,1$, or 2$)$ for these five items.

The remainder of The CSDC assesses the thirty different post traumatic symptoms on five dimensions- 1) Reexperiencing, 2) Avoidance, 3) Numbing and Dissociation, 4) Increased Arousal, 5) Impairment in Functioning. The items that measure these five dimensions are-

1) Reexperiencing- Items $1,3,12,19,22,23,25$

2) Avoidance- Items 5, 14, 21, 28, 30

3) Numbing and Dissociation- Items 4, 7, 9, 16, 18, 20, 26, 27

4) Increased Arousal- Items 2, 6, 8, 13, 17, 29

5) Impairment in Function- Items 10, 11, 15, 24

The score for each dimension is calculated by adding the responses for each item in that dimension. The total Post Traumatic Symptom Score is calculated by adding the responses for all 30 items.

\section{SCORES}

1) Immediate Response Score:

2) Post Traumatic Symptom Scores:

1) Reexperiencing:

2) Avoidance:

3) Numbing and Dissociation:

4) Increased Arousal:

5) Impairment in Functioning:

6) Total Post Traumatic Symptom Score: 
Height $\mathrm{cm}$ End Time

Weight $\mathrm{kg}$

\section{Treadmill Protocol}

Note: During the initial 3 minutes, Target heart rate should be reached and maintained throughout the exercise. $($ THR $=220$ - age $x$.70).

Gradually increase speed until participant reaches comfortable walking speed. This speed will be maintained the entire time. Next, increase grade until target heart rate is reached. Adjust grade to maintain THR as needed. This needs to be finished by the start of minute 3 (Shaded area)

Target HR

\begin{tabular}{|l|l|l|l|l|}
\hline Minute & Speed & Grade & HR & RPE (Every 3 min) \\
\hline $0: 00-0: 59$ & $1.5 \mathrm{mph}$ & $3 \%$ & & \\
\hline $1: 00-1: 59$ & & & & \\
\hline $2: 00-2: 59$ & & & & \\
\hline $3: 00-3: 59$ & & & & \\
& & & & \\
\hline
\end{tabular}




\begin{tabular}{|c|c|c|c|c|}
\hline 4:00-4:59 & & & & \\
\hline Minute & Speed & Grade & HR & RPE \\
\hline 5:00-5:59 & & & & \\
\hline 6:00-6:59 & & & & \\
\hline 7:00-7:59 & & & & \\
\hline 8:00-8:59 & & & & \\
\hline 9:00-9:59 & & & & \\
\hline $10: 00-10: 59$ & & & & \\
\hline $11: 00-11: 59$ & & & & \\
\hline $12: 00-12: 59$ & & & & \\
\hline $13: 00-13: 59$ & & & & \\
\hline $14: 00-14: 59$ & & & & \\
\hline & & & & \\
\hline
\end{tabular}




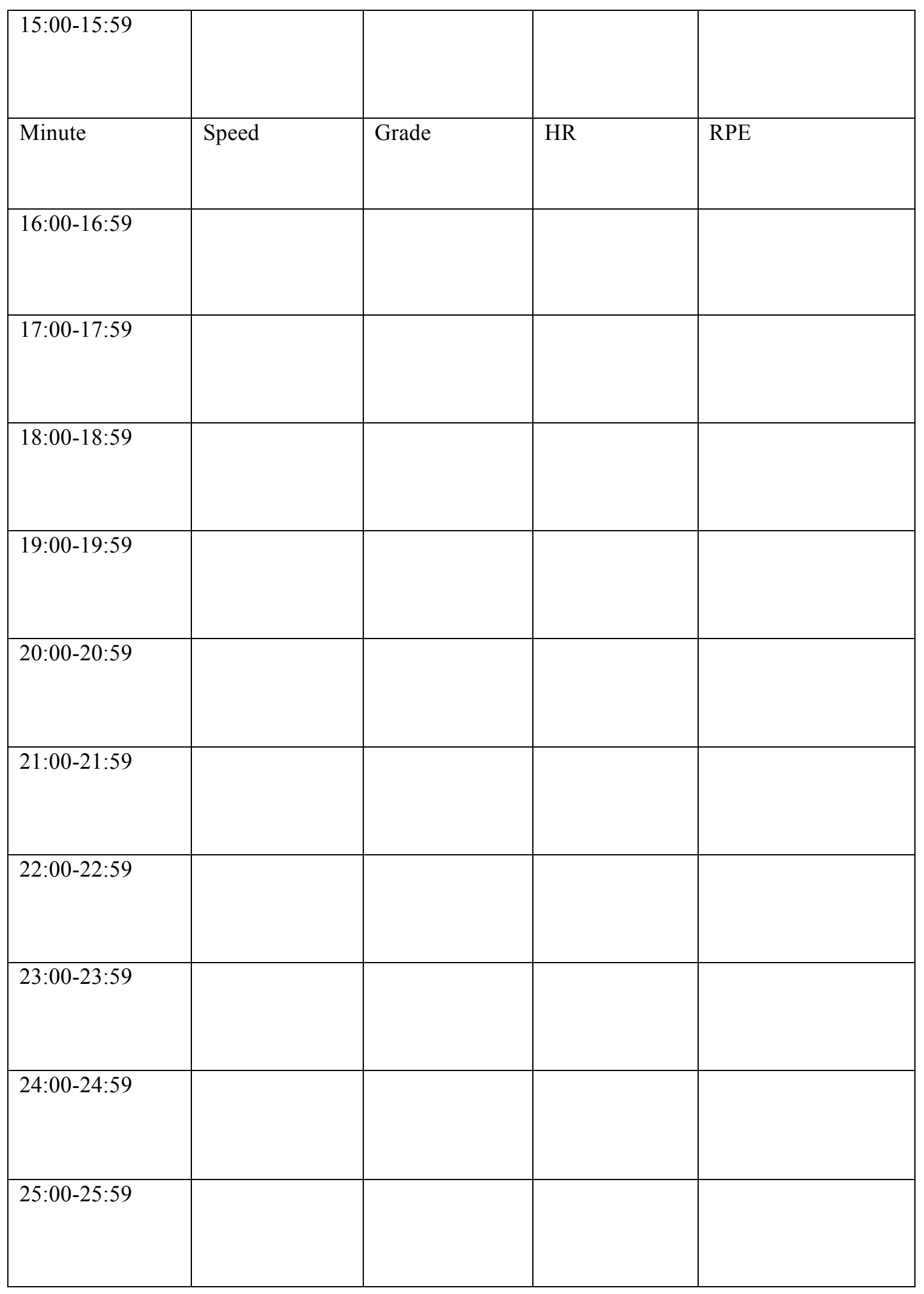




\begin{tabular}{|l|l|l|l|l|}
\hline 26:00-26:59 & & & & \\
\hline Minute & Speed & Grade & HR & RPE \\
\hline $27: 00-27: 59$ & & & & \\
\hline $28: 00-28: 59$ & & & & \\
\hline $29: 00-29: 59$ & & & & \\
\hline $30: 00-30: 59$ & & & & \\
\hline $31: 00-31: 59$ & & & & \\
\hline Cool Down & $2.0-2.5$ mph & $0 \%$ Grade & & \\
\hline $32: 00-32: 59$ & & & & \\
\hline & & & & \\
\hline
\end{tabular}




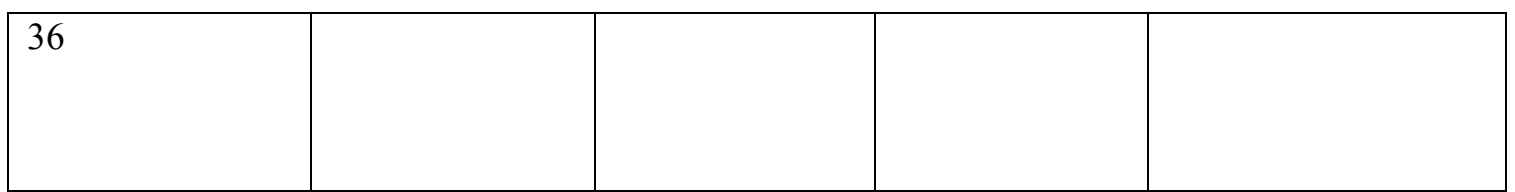

Comments: 
Appendix F

RPE Scale for Kids

\section{RPE Scale for Kids!}

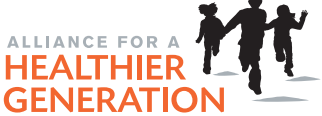

RPE stands for Relative Perceived Exertion. Relative Perceived Exertion means, "How hard to I feel I am exercising?" It is a tool you use to tell others how your body is feeling when you exercise. Kids who exercise in the yellow zone are getting moderate to vigorous exercise. That means your body is getting the right amount to be healthy! You should get 60 minutes of exercise in the yellow zone every day.

\begin{tabular}{|c|c|c|c|}
\hline $\begin{array}{l}\text { My } \\
\text { number... }\end{array}$ & $\begin{array}{l}\text { My } \\
\text { face... }\end{array}$ & This is what I may be thinking..... & This is what my body may be doing.... \\
\hline & & This exercise is the same as resting. & $\begin{array}{l}\text { I am getting ready to exercise, } \\
\text { but I don't feel different yet. }\end{array}$ \\
\hline & & This exercise isn't hard. & $\begin{array}{l}\text { I am getting a little warm. } \\
\text { I can still talk normally. }\end{array}$ \\
\hline & & $\begin{array}{l}\text { I am just beginning to feel } \\
\text { like I am exercising. }\end{array}$ & $\begin{array}{l}\text { I am feeling like my } \\
\text { body is warming up. }\end{array}$ \\
\hline & & $\begin{array}{l}\text { I am starting to feel like I am } \\
\text { exercising. I feel good! }\end{array}$ & $\begin{array}{l}\text { I can almost talk in a regular } \\
\text { voice, but it is getting harder. }\end{array}$ \\
\hline & & $\begin{array}{l}\text { This exercise is a good workout! } \\
\text { I am really working hard. }\end{array}$ & $\begin{array}{l}\text { My cheeks are getting pink. } \\
\text { I am getting a little sweaty. }\end{array}$ \\
\hline & & $\begin{array}{l}\text { I am exercising more than I thought. } \\
\text { It is getting hard to do. }\end{array}$ & $\begin{array}{l}\text { I feel like talking is getting harder. } \\
\text { I have to stop sometimes for air. }\end{array}$ \\
\hline & & $\begin{array}{l}\text { This is pretty hard. I can exercise a } \\
\text { little bit longer, then I'll stop. }\end{array}$ & $\begin{array}{l}\text { I am getting really sweaty. } \\
\text { My body is hot! }\end{array}$ \\
\hline & & $\begin{array}{l}\text { This exercise is really hard, } \\
\text { but I'm not ready to quit. }\end{array}$ & I can talk a little, but not too much \\
\hline & & $\begin{array}{l}\text { I need a break from this } \\
\text { very hard exercise. }\end{array}$ & $\begin{array}{l}\text { My face looks red. I feel like } \\
\text { I need to stop. }\end{array}$ \\
\hline & & $\begin{array}{l}\text { I'm exercising too much! My body } \\
\text { is making me stop now! }\end{array}$ & $\begin{array}{l}\text { My heart is beating very fast } \\
\text { and strong. I can't talk. }\end{array}$ \\
\hline
\end{tabular}

